\title{
R-OPERATOR, CO-PRODUCT AND HAAR-MEASURE FOR THE MODULAR DOUBLE OF $U_{q}(\mathfrak{s l}(2, \mathbb{R}))$
}

\author{
by
}

A.G. Bytsko and J. Teschner

\begin{abstract}
A certain class of unitary representations of $U_{q}(\mathfrak{s l}(2, \mathbb{R}))$ has the property of being simultanenously a representation of $U_{\tilde{q}}(\mathfrak{s l}(2, \mathbb{R}))$ for a particular choice of $\tilde{q}(q)$. Faddeev has proposed to unify the quantum groups $U_{q}(\mathfrak{s l}(2, \mathbb{R}))$ and $U_{\tilde{q}}(\mathfrak{s l}(2, \mathbb{R}))$ into some enlarged object for which he has coined the name "modular double".

We study the R-operator, the co-product and the Haar-measure for the modular double of $U_{q}(\mathfrak{s l}(2, \mathbb{R}))$ and establish their main properties. In particular it is shown that the Clebsch-Gordan maps constructed in [PT2] diagonalize this R-operator.

MSC:

Keywords: non-compact quantum groups, modular double
\end{abstract}

\section{Introduction}

Quantum groups have become an indispensable tool in many areas of mathematical physics and mathematics. In a broad class of quantum theoretical models it has turned out that finding a relation to a quantum group is the key for obtaining exact information about the spectrum or the correlation functions.

So far most of the vast amount of work devoted to quantum group theory and their physical applications was concerned with quantum groups that can be studied in a purely algebraic manner. This is the case e.g. if the relevant representations are highest weight representations, as is often assumed.

However, in physical applications to quantum theoretical models the choice of a scalar product on the space of states usually determines the hermiticity relations for the representatives of the quantum group generators. In many cases like those corresponding to the so-called non-compact quantum groups it turns out that the corresponding unitary representations are always infinite-dimensional and generically neither of highest nor lowest weight type. In order to exploit the information provided by the appearance of such a quantum group it is clearly important to have efficient mathematical tools for analyzing the corresponding representation theory.

Unfortunately there are comparatively few results about the representation theory of non-compact quantum groups. This seems to be an important obstacle for making 
further progress in many quantum integrable models. Nevertheless there is an interesting example that was first studied independently in [PT1], [F2] and [PT2]. These references were considering a particular class of infinite dimensional unitary representations of $U_{q}(\mathfrak{s l}(2, \mathbb{R}))$. This class of representations, henceforth denoted $\mathscr{P}_{s}, s \in \mathbb{R}$, was the also first to be used in a concrete physical application: Understanding the tensor products of the above-mentioned representations was crucial for obtaining exact results on the quantum Liouville theory [PT1][1].

A rather remarkable duality phenomenon was observed in [F1], [PT1] and [F2]. The representations in question are simultaneously representations of the two quantum groups $U_{q}\left(\mathfrak{s l}_{2}\right)$ and $U_{\tilde{q}}\left(\mathfrak{s l}_{2}\right)$ with deformation parameters $q=e^{\pi i b^{2}}$ and $\tilde{q}=e^{\frac{\pi i}{b^{2}}}$ respectively. This duality turns out to be deeply related to the quantum field theoretical self-duality of Liouville theory under the change of the coupling constant $b$ into $b^{-1}$ [1]. Moreover, it is this duality under $b \rightarrow b^{-1}$ that allows one to cover the socalled strong-coupling regime where $|b|=1$ by analytic continuation of the results for real values of $b[$ PT1 $][$ FKV $][$ T $][$ FK2]

The results of the present paper clarify the origin of this phenomenon to a certain extent. Given the operators X representing one of the two algebras, say $U_{q}\left(\mathfrak{s l}_{2}\right)$, one may obtain the representatives of the second algebra $U_{\tilde{q}}\left(\mathfrak{s l}_{2}\right)$ as nonpolynomial operator functions of the operators $X$. This is found to be consistent with the respective co-products. In particular, restricting attention to only one of the two algebras does not lead to any degeneracy as is sometimes suggested in the literature.

Faddeev has proposed to unite the quantum groups $U_{q}\left(\mathfrak{s l}_{2}\right)$ and $U_{\tilde{q}}\left(\mathfrak{s l}_{2}\right)$ into some enlarged object for which he has coined the name "modular double". The proposal of [F2] as refined in [KLS] amounts to defining it as the product of $\hat{U}_{q}\left(\mathfrak{s l}_{2}\right)$ and $\hat{U}_{\tilde{q}}\left(\mathfrak{s l}_{2}\right)$, where, roughly speaking, $\hat{U}_{q}\left(\mathfrak{s l}_{2}\right)$ is obtained from $U_{q}\left(\mathfrak{s l}_{2}\right)$ by adjoining a sign to the center generated by the Casimir of $U_{q}\left(\mathfrak{s l}_{2}\right)$. We feel that this definition for the modular double has a disadvantage, though. Most representations of $\hat{U}_{q}\left(\mathfrak{s l}_{2}\right) \otimes \hat{U}_{\tilde{q}}\left(\mathfrak{s l}_{2}\right)$ are simply tensor products of representations of the two factors. The representations $\mathscr{P}_{s}$ on the contrary are distinguished by the fact that they do not factorize as a tensor product of representations for $U_{q}\left(\mathfrak{s l}_{2}\right)$ and $U_{\tilde{q}}\left(\mathfrak{s l}_{2}\right)$. This is what makes the duality under $b \rightarrow b^{-1}$ a nontrivial statement. Since the category formed by the representations $\mathscr{P}_{s}$ is closed under tensor products [PT2] it seems natural to look for the group-like object that contains the interesting representations $\mathscr{P}_{s}$ only.

We therefore propose to look for a definition of the "modular double" that excludes the representations of $U_{q}\left(\mathfrak{s l}_{2}\right) \otimes U_{\tilde{q}}\left(\mathfrak{s l}_{2}\right)$ which factorize trivially. This definition should still capture the duality phenomenon mentioned above. As we have indicated, this naturally leads us to consider nonpolynomial functions of the generators. The basic objects underlying our approach to the modular double will be an algebra $\mathscr{A}$ of bounded operators, a coproduct $\Delta$ on $\mathscr{A}$, an invariant integration (Haar-measure) on $\mathscr{A}$ and the R-operator proposed in [F2]. We are going to establish the main properties satisfied by these objects, which are all self-dual under $b \rightarrow b^{-1}$. 
The algebra $\mathscr{A}$ can be thought of as being generated from operators that represent $U_{q}\left(\mathfrak{s l}_{2}\right)$ in a similar (in fact, closely related) way as the algebra of bounded operators on $L^{2}(\mathbb{R})$ is related to the usual quantum mechanical position and momentum operators $x$ and $p$. Our point of view is inspired by the one of Woronowicz [W1], which has stimulated the development of a theory for noncompact quantum groups in a $C^{*}$ algebraic framework, see e.g. $[\mathbf{K V}]$ and references therein. However, although we believe that our results represent substantial progress towards a proof that the modular double fits into such a $C^{*}$-algebraic framework, it was not our aim to actually carry out such a proof here.

We also clarify the relation between the R-operator proposed in [F2] and the calculus of Clebsch-Gordan and Racah-Wigner-coefficients of [PT2]. Establishing this relation is important for the following reason. In [T] it was shown that certain families of representations of the Virasoro algebra and of the quantum group $U_{q}(\mathfrak{s l}(2, \mathbb{R}))$ behave equivalently under the respective product operations (fusion and tensor product). Together with the results of the present paper it follows that the respective braiding operations are equivalent as well.

To be specific we will mostly consider the case that the deformation parameter is of the form $q=e^{\pi i b^{2}}$, where $b \in(0,1)$. However, our results will carry over to the "strong coupling regime" $|b|=1$, see the remarks in Subsection 2.11.

\section{Definitions and main results}

2.1. Star algebra $U_{q}(\mathfrak{s l}(2, \mathbb{R})) \cdot-U_{q}(\mathfrak{s l}(2, \mathbb{R}))$ is a Hopf-algebra with generators: $E, \quad F, \quad K, K^{-1}$;

relations: $\quad K E=q E K, \quad K F=q^{-1} F K, \quad[E, F]=\frac{K^{2}-K^{-2}}{q-q^{-1}}$;

star-structure: $\quad K^{*}=K, \quad E^{*}=E, \quad F^{*}=F$.

The center of $U_{q}(\mathfrak{s l}(2, \mathbb{R}))$ is generated by the $q$-Casimir

$$
C=F E+\frac{q K^{2}+q^{-1} K^{-2}-2}{\left(q-q^{-1}\right)^{2}} .
$$

Compared to the definition used in [PT2] we have redefined $F \rightarrow-F$. This will allow us to realize $F$ by positive operators.

2.2. The representations $\mathscr{P}_{s}$ of $U_{q}(\mathfrak{s l}(2, \mathbb{R}))$. - In the present paper we will study a one-parameter class $\mathscr{P}_{s}, s \in \mathbb{R}$, of representations of $U_{q}(s l(2, \mathbb{R}))$. They are constructed as follows: The representation will be realized on the space $\mathscr{P}_{s}$ of entire analytic functions $f(x)$ that have a Fourier-transform $\tilde{f}(\omega)$ which is meromorphic in $\mathbb{C}$ with the possible poles contained in

$$
\mathscr{S}_{s} \equiv\left\{ \pm \omega=s+i\left(\frac{Q}{2}+n b+m b^{-1}\right), n, m \in \mathbb{Z}^{\geq 0}\right\}
$$


where $Q=b+b^{-1}$. The representation of $U_{q}(\mathfrak{s l}(2, \mathbb{R}))$ on $\mathscr{P}_{s}$ is then defined by choosing the representatives $\pi_{s}(X)$ for $X=E, F, K$ to be the following finite difference operators

$$
\begin{aligned}
& \pi_{s}(E) \equiv \mathrm{E}_{s} \equiv e^{+\pi b \times} \frac{\cosh \pi b(\mathrm{p}-s)}{\sin \pi b^{2}} e^{+\pi b \times} \\
& \pi_{s}(F) \equiv \mathrm{F}_{s} \equiv e^{-\pi b \times} \frac{\cosh \pi b(\mathrm{p}+s)}{\sin \pi b^{2}} e^{-\pi b \times}
\end{aligned}
$$

where $\mathrm{p}$ and $\mathrm{x}$ are self-adjoint operators satisfying $[\mathrm{p}, \mathrm{x}]=(2 \pi i)^{-1}$. By embedding $\mathscr{P}_{s}$ as a dense subspace into the Hilbert space $L^{2}(\mathbb{R})$ one obtains a unitary representation of $U_{q}(\mathfrak{s l}(2, \mathbb{R}))$ generated from the self-adjoint operators $\mathrm{E}, \mathrm{F}$ and $\mathrm{K}[\mathbf{S}]$.

2.3. The representations $\mathscr{H}$ and $\mathscr{K}$ of $U_{q}(s l(2, \mathbb{R}))$. - We will find it convenient to formulate our results in a "universal" setting. Let us define $\mathscr{K} \equiv L^{2}(\mathbb{R} \times \mathbb{R})$. The algebra $\mathscr{B}(\mathscr{K})$ of bounded operators on $\mathscr{K}$ is generated by two pairs $\left(\mathrm{x}_{i}, \mathrm{p}_{i}\right), i=1,2$ satisfying $\left[\mathrm{p}_{i}, \mathrm{x}_{i}\right]=(2 \pi i)^{-1}$. The action of $U_{q}(\mathfrak{s l}(2, \mathbb{R}))$ on $\mathscr{K}$ is defined by

$$
\begin{aligned}
& \pi_{\mathscr{K}}(E) \equiv \mathrm{E}=e^{\pi b\left(\mathrm{x}_{1}-\mathrm{x}_{2}\right)} \frac{\cosh \pi b \mathrm{p}_{2}}{\sin \pi b^{2}} e^{\pi b\left(\mathrm{x}_{1}-\mathrm{x}_{2}\right)}, \\
& \pi_{\mathscr{K}}(F) \equiv \mathrm{F}=e^{\pi b\left(\mathrm{x}_{2}-\mathrm{x}_{1}\right)} \frac{\cosh \pi b \mathrm{p}_{1}}{\sin \pi b^{2}} e^{\pi b\left(\mathrm{x}_{2}-\mathrm{x}_{1}\right)},
\end{aligned}
$$

This representation of $U_{q}(\mathfrak{s l}(2, \mathbb{R}))$ on $\mathscr{K}$ is reducible: The operator $\mathrm{s}=\frac{1}{2}\left(\mathrm{p}_{1}+\mathrm{p}_{2}\right)$ commutes with $\mathrm{E}, \mathrm{F}, \mathrm{K}$ and determines the representation of the Casimir via

$$
\mathrm{C} \equiv \pi_{\mathscr{K}}(C)=\frac{\cosh ^{2} \pi b \mathrm{~s}}{\sin ^{2} \pi b^{2}} .
$$

The action of (2.5) on an eigenspace of s reduces to the action (2.4) on $\mathscr{P}_{s}$ upon identification $\mathrm{p}=\frac{1}{2}\left(\mathrm{p}_{1}-\mathrm{p}_{2}\right)$ and $\mathrm{x}=\mathrm{x}_{1}-\mathrm{x}_{2}$. This means that $\mathscr{K}$ decomposes into the representations $\mathscr{P}_{s}$ as follows:

$$
\mathscr{K} \simeq \int_{\mathbb{R}}^{\oplus} d s \mathscr{P}_{s}
$$

The representations $\mathscr{P}_{s}$ and $\mathscr{P}_{-s}$ are unitarily equivalent [PT2]: There exists a unitary operator $\mathrm{J}_{s}: \mathscr{P}_{s} \rightarrow \mathscr{P}_{-s}$ such that $\mathrm{X}_{-s} \mathrm{~J}_{s}=\mathrm{J}_{s} \mathrm{X}_{s}$ for all $X \in U_{q}(\mathfrak{s l}(2, \mathbb{R}))$. The operator $\mathrm{J}_{s}$ defines an operator $\mathrm{J}: \mathscr{K} \rightarrow \mathscr{K}$ if one considers the operator function $\mathrm{J} \equiv \mathrm{J}_{\mathrm{s}}$.

It will sometimes be convenient to consider instead of $\mathscr{K}$ a space $\mathscr{H}$ in which $\mathscr{P}_{s}$ and $\mathscr{P}_{-s}$ are identified:

$$
\mathscr{H}=\{v \in \mathscr{K} ;(\mathrm{id}-\mathrm{J}) v=0\} .
$$

$\mathscr{H}$ is of course isomorphic to $\int_{\mathbb{R}^{+}}^{\oplus} d s \mathscr{P}_{s}$. 
2.4. Operator functions of $E, F, K$. - It is important to also consider nonpolynomial functions of the operators $\mathrm{E}, \mathrm{F}, \mathrm{K}$. Let us first note that standard functional calculus for positive selfadjoint operators allows one to consider complex powers of the generators such as $E^{\gamma}, \gamma \in \mathbb{C}$. The following result offers a partial explanation for the phenomenon of modular duality.

Lemma 1. - (i) The operators $\tilde{\mathrm{E}}_{s}, \tilde{\mathrm{F}}_{s}, \tilde{\mathrm{K}}_{s}$ obtained by replacing $b \rightarrow b^{-1}$ in (2.4),

$$
\begin{aligned}
& \tilde{\mathrm{E}}_{s} \equiv e^{+\pi b^{-1} \times} \frac{\cosh \pi b^{-1}(\mathrm{p}-s)}{\sin \pi b^{-2}} e^{+\pi b^{-1} \times} \\
& \tilde{\mathrm{F}}_{s} \equiv e^{-\pi b^{-1} \times} \frac{\cosh \pi b^{-1}(\mathrm{p}+s)}{\sin \pi b^{-2}} e^{-\pi b^{-1} \times}
\end{aligned}
$$

generate a representation of $U_{\tilde{q}}(s l(2, \mathbb{R}))$ with $\tilde{q}=e^{\pi i b^{-2}}$. The generators $\tilde{\mathrm{E}}_{s}, \tilde{\mathrm{F}}_{s}, \tilde{\mathrm{K}}_{s}$ commute with the operators $\mathrm{E}_{s}, \mathrm{~F}_{s}, \mathrm{~K}_{s}$ on $\mathscr{P}_{s}$.

(ii) For $\gamma=b^{-2}$ we have

$$
\begin{aligned}
& \left(\sin \left(\pi b^{2}\right) \mathrm{E}_{s}\right)^{\gamma}=\sin \left(\pi b^{-2}\right) \tilde{\mathrm{E}}_{s}, \quad \mathrm{~K}_{s}^{\gamma}=\tilde{\mathrm{K}}_{s} . \\
& \left(\sin \left(\pi b^{2}\right) \mathrm{F}_{s}\right)^{\gamma}=\sin \left(\pi b^{-2}\right) \tilde{\mathrm{F}}_{s},
\end{aligned}
$$

Being operator functions of $\mathrm{E}_{s}, \mathrm{~F}_{s}, \mathrm{~K}_{s}$, the operators $\tilde{\mathrm{E}}_{s}, \tilde{\mathrm{F}}_{s}, \tilde{\mathrm{K}}_{s}$ do not commute with $\mathrm{E}_{s}, \mathrm{~F}_{s}, \mathrm{~K}_{s}$ in the usual sense (commutativity of the spectral projections).

We shall now define an algebra of bounded operators that can be considered as operator functions of E, F, K. To begin with, let $\mathscr{O}_{s}, s \in \mathbb{R}$ be a family of bounded operators on $L^{2}(\mathbb{R})$ such that

$$
\sup _{s \in \mathbb{R}}\left\|\mathscr{O}_{s}\right\|<\infty .
$$

A bounded operator $\mathscr{O}$ on $\mathscr{K}$ can be defined for each such family $\left(\mathscr{O}_{s}\right)_{s \in \mathbb{R}}$ by means of (2.7). These operators $\mathscr{O}$ form a subalgebra $\mathscr{B}_{0}$ of the algebra of all bounded operators on $\mathscr{H}$. Let $\mathscr{B}$ be the $C^{*}$ subalgebra obtained as the completion of $\mathscr{B}_{0}$ w.r.t. the operator norm. This algebra can be thought of as being generated from the unbounded elements $\mathrm{p}, \mathrm{x}, \mathrm{s}$.

However, there is no canonical way to define $\operatorname{sgn}(\mathrm{s})$ as a function of $E, F, K$. The center of the algebra of bounded operators generated from E, F, K should be generated from operator functions of the Casimir, or equivalently $|s|$, cf. eqn. (2.6). This is closely related to the fact that the representations $\mathscr{P}_{s}$ and $\mathscr{P}_{-s}$ are unitarily equivalent. Elements of the "true" algebra $\mathscr{A} \subset \mathscr{B}$ should therefore commute with the operator J which establishes the equivalence between $\mathscr{P}_{s}$ and $\mathscr{P}_{-s}$,

$$
\mathscr{A} \equiv\left\{\mathrm{O} \in \mathscr{B} ; \mathrm{J}^{-1} \mathrm{OJ}=\mathrm{O}\right\} .
$$

This amounts to considering only those elements of $\mathscr{B}$ that leave $\mathscr{H}$ invariant. 
2.5. The Hopf algebra structure. - A co-product is defined on $U_{q}(\mathfrak{s l}(2, \mathbb{R}))$ via

$$
\begin{aligned}
& \Delta(E)=E \otimes K+K^{-1} \otimes E, \\
& \Delta(F)=F \otimes K+K^{-1} \otimes F,
\end{aligned}
$$

In the following we shall adopt the convention to denote

$$
\Delta(\mathrm{X}) \equiv\left(\pi_{\mathscr{H}} \otimes \pi_{\mathscr{H}}\right) \circ \Delta(X) \quad \text { for } \quad X \in \mathscr{U}_{q}(\mathfrak{s l}(2, \mathbb{R})) .
$$

It follows from [PT2, Theorem 2] that $\Delta(\mathrm{E}), \Delta(\mathrm{F})$ and $\Delta(\mathrm{K})$ are self-adjoint and positive and therefore generate a representation of $U_{q}(\mathfrak{s l}(2, \mathbb{R}))$ on $\mathscr{H} \otimes \mathscr{H}$. The following Lemma proven in Section 3 establishes consistency of the co-product with modular duality:

Lemma 2. - The co-product (2.11)(2.12) acts on the dual part of the modular double as follows.

$$
\begin{aligned}
& \Delta(\tilde{\mathrm{E}})=\tilde{\mathrm{E}} \otimes \tilde{\mathrm{K}}+\tilde{\mathrm{K}}^{-1} \otimes \tilde{\mathrm{E}}, \\
& \Delta(\tilde{\mathrm{F}})=\tilde{\mathrm{F}} \otimes \tilde{\mathrm{K}}+\tilde{\mathrm{K}}^{-1} \otimes \tilde{\mathrm{F}}, \quad \Delta(\tilde{\mathrm{K}})=\tilde{\mathrm{K}} \otimes \tilde{\mathrm{K}} .
\end{aligned}
$$

A representation of the co-product on the algebra $\mathscr{A}$ can be defined by means of the Clebsch-Gordan maps defined in [ [PT2]. These maps yield a three parameter family of maps $\mathrm{C}\left[s_{3} \mid s_{2}, s_{1}\right]: \mathscr{P}_{s_{2}} \otimes \mathscr{P}_{s_{1}} \rightarrow \mathscr{P}_{s_{3}}$ that satisfy the intertwining property

$$
\mathrm{C}\left[s_{3} \mid s_{2}, s_{1}\right] \circ\left(\pi_{s_{2}} \otimes \pi_{s_{1}}\right) \circ \Delta(X)=\pi_{s_{3}}(X) \circ \mathrm{C}\left[s_{3} \mid s_{2}, s_{1}\right]
$$

and extends to a two-parameter family of unitary operators $\mathrm{C}\left[s_{2}, s_{1}\right]: L^{2}\left(\mathbb{R}^{2}\right) \rightarrow \mathscr{H}$. Let us introduce the operators $\mathrm{s}_{1}=\mathrm{id} \otimes \mathrm{s}, \mathrm{s}_{2}=\mathrm{s} \otimes \mathrm{id}$ on $\mathscr{H} \otimes \mathscr{H}$ respectively. The identification (2.7) allows us to consider $\mathrm{C}\left[\mathrm{s}_{2}, \mathrm{~s}_{1}\right]$ as a unitary operator

$$
\mathrm{C}: \mathscr{H} \otimes \mathscr{H} \rightarrow \mathscr{H} \otimes \mathscr{H}_{\text {spec }}^{(2)} \otimes \mathscr{H}_{\text {spec }}^{(1)}
$$

where the operators $\mathrm{s}_{i}, i=1,2$ are realized on the spaces $\mathscr{H}_{\text {spec }}^{(i)} \simeq L^{2}\left(\mathbb{R}^{+}\right)$as multiplication operators.

For each element $\mathrm{X} \in \mathscr{A}$ we may now define $\Delta(\mathrm{X})$ by

$$
\Delta(\mathrm{X}) \equiv \mathrm{C}^{\dagger} \circ(\mathrm{X} \otimes \mathrm{id} \otimes \mathrm{id}) \circ \mathrm{C} .
$$

Since $\mathrm{C}$ is unitary and $\mathrm{X}$ is bounded we clearly have boundedness of $\Delta(\mathrm{X}): \mathscr{H} \otimes \mathscr{H} \rightarrow$ $\mathscr{H} \otimes \mathscr{H}$.

Theorem 1. - The coproduct $\Delta$ is coassociative on $\mathscr{A}$, i.e.

$$
(\mathrm{id} \otimes \Delta) \circ \Delta(\mathrm{X})=(\Delta \otimes \mathrm{id}) \circ \Delta(\mathrm{X}) \text { for any } \mathrm{X} \in \mathscr{A} \text {. }
$$

The antipode consistent with (2.11) is defined as an anti-automorphism of $U_{q}(\mathfrak{s l}(2, \mathbb{R}))$ such that

$$
\sigma(K)=K^{-1}, \quad \sigma(E)=-q E, \quad \sigma(F)=-q^{-1} F .
$$


The action of the antipode on nonpolynomial functions of $\mathrm{E}, \mathrm{F}$ and $\mathrm{K}$ can be introduced by means of

$$
\sigma(\mathrm{p})=-\mathrm{p}, \quad \sigma(\mathrm{s})=-\mathrm{s}, \quad \sigma(\mathrm{x})=\mathrm{x}+\frac{i}{2} Q .
$$

The fact that $\mathrm{x}$ is shifted by an imaginary amount means that $\sigma$ is not defined on all of $\mathscr{A}$. This unboundedness of the antipode is not unexpected [KV].

2.6. The Haar-measure. - Let us first note that the decomposition (2.7) induces a family of projections $\pi_{s}: \mathscr{A} \rightarrow \mathscr{B}\left(L^{2}(\mathbb{R})\right)$. We shall often use the shorthand notation $\mathrm{O}_{s} \equiv \pi_{s}(\mathrm{O})$.

Definition 1. - Define linear functionals $\mathrm{h}_{1}$ and $\mathrm{h}_{\mathrm{r}}$ on dense subsets $\mathscr{A}_{\mathrm{h}}^{\mathrm{l}}$ and $\mathscr{A}_{\mathrm{h}}^{\mathrm{r}}$ of $\mathscr{A}$ respectively by

$$
\begin{aligned}
& \mathrm{h}_{1}(\mathrm{O})=\int_{0}^{\infty} d \mathrm{~m}(s) \operatorname{Tr}\left(e^{-2 \pi Q \mathrm{p}} \mathrm{O}_{s}\right), \\
& \mathrm{h}_{\mathrm{r}}(\mathrm{O})=\int_{0}^{\infty} d \mathrm{~m}(s) \operatorname{Tr}\left(e^{+2 \pi Q \mathrm{p}} \mathrm{O}_{s}\right),
\end{aligned}
$$

where the measure $m$ is defined by

$$
d \mathrm{~m}(s) \equiv 4 \sinh 2 \pi b s \sinh 2 \pi b^{-1} s d s .
$$

Theorem 2. - (i) The Haar-measures $\mathrm{h}_{1}$ and $\mathrm{h}_{\mathrm{r}}$ are left and right invariant respectively,

$$
\begin{aligned}
& \left(\mathrm{id} \otimes \mathrm{h}_{1}\right) \circ \Delta(\mathrm{O})=\mathrm{h}_{1}(\mathrm{O}) \mathrm{id}, \\
& \left(\mathrm{h}_{\mathrm{r}} \otimes \mathrm{id}\right) \circ \Delta(\mathrm{O})=\mathrm{h}_{\mathrm{r}}(\mathrm{O}) \mathrm{id},
\end{aligned}
$$

where we assume $\mathrm{O}$ to be taken from the respective domains of definition.

(ii) For any $\mathrm{X} \in U_{q}(\mathfrak{s l}(2, \mathbb{R}))$, the Haar-measures satisfy, respectively

$$
\begin{aligned}
& \mathrm{h}_{1}\left(\operatorname{ad}_{X}^{1} \mathrm{O}\right)=\mathrm{h}_{1}(\mathrm{O}) \varepsilon(\mathrm{X}), \\
& \mathrm{h}_{\mathrm{r}}\left(\operatorname{ad}_{\mathrm{X}}^{\mathrm{r}} \mathrm{O}\right)=\mathrm{h}_{\mathrm{r}}(\mathrm{O}) \varepsilon(\mathrm{X}),
\end{aligned}
$$

where $\varepsilon(X)$ is the co-unit, and the left and right q-adjoint actions are defined as $\operatorname{ad}_{\mathrm{X}}^{\mathrm{l}}(Y)=\sum_{i} X_{i}^{\prime} Y \sigma\left(X_{i}^{\prime \prime}\right)$ and $\operatorname{ad}_{\mathrm{X}}^{\mathrm{r}}(Y)=\sum_{i} \sigma\left(X_{i}^{\prime}\right) Y X_{i}^{\prime \prime}$ if $\Delta(X)=\sum_{i} X_{i}^{\prime} \otimes X_{i}^{\prime \prime}$.

Remark 1. - We believe that the triple $(\mathscr{A}, \Delta, h)$ that we have defined above constitutes a somewhat more satisfactory definition of the modular double, although more work is needed to show that it fits into the axiomatics for noncompact quantum groups of [KV]. The self-duality under $b \rightarrow b^{-1}$ is manifest in this formulation.

It also becomes clear that the modular double can not be considered as a deformation of a classical group: The Haar-measure has no classical limit $b \rightarrow 0$ due to the factor $Q=b+b^{-1}$ that appears in the definition of $\mathrm{h}$. 
2.7. The R-operator. - To begin with, we introduce the special function $g_{b}(x)$ that will be used to define the R-operator. It may be defined via (recall that $Q=b+b^{-1}$ )

$$
\log g_{b}(x)=-\int_{\mathbb{R}+i 0} \frac{d t}{t} \frac{e^{\frac{t Q}{2}} x^{\frac{t}{2 \pi i b}}}{\left(1-e^{b t}\right)\left(1-e^{t / b}\right)} .
$$

Let us furthermore introduce an anti-self-adjoint element $\mathrm{H}$ such that $K=q^{H}$. Define

$$
\mathrm{R}=q^{\mathrm{H} \otimes \mathrm{H}} g_{b}\left(4\left(\sin \pi b^{2}\right)^{2} \mathrm{E} \otimes \mathrm{F}\right) q^{\mathrm{H} \otimes \mathrm{H}},
$$

where $\mathrm{H} \equiv \pi_{\mathscr{H}}(H)$. As we will explain below (see Corollary 2), R coincides with the R-operator proposed by L. Faddeev in [F2]. Notice that the property (3.5) implies that $\left|g_{b}(x)\right|=1$ for $x \in \mathbb{R}^{+}$. This makes $\mathrm{R}$ manifestly unitary.

Theorem 3. - The operator $\mathrm{R}$ has the following properties:

(i) $\mathrm{R} \Delta(\mathrm{X})=\Delta^{\prime}(\mathrm{X}) \mathrm{R}$

(ii) $\quad($ id $\otimes \Delta) R=R_{13} R_{12}, \quad(\Delta \otimes$ id $) R=R_{13} R_{23}$,

(iii) $\quad(\sigma \otimes$ id $) \mathrm{R}=\mathrm{R}^{-1}, \quad($ id $\otimes \sigma) \mathrm{R}=\mathrm{R}^{-1}, \quad(\sigma \otimes \sigma) \mathrm{R}=\mathrm{R}$.

Remark 2. - The R-operator allows us to introduce the braiding of tensor products of the representations $\mathscr{P}_{s}$. Specifically, let the operator B: $\mathscr{P}_{s_{2}} \otimes \mathscr{P}_{s_{1}} \rightarrow \mathscr{P}_{s_{1}} \otimes \mathscr{P}_{s_{2}}$ be defined by $\mathrm{B}_{s_{2}, s_{1}} \equiv \mathrm{PR}_{s_{2}, s_{1}}$, where $\mathrm{P}$ is the operator that permutes the two tensor factors. Property (i) from Theorem 3 implies as usual that $\mathrm{B}_{s_{2}, s_{1}} \circ \Delta(\mathrm{X})=\Delta(\mathrm{X}) \circ \mathrm{B}_{s_{2}, s_{1}}$.

2.8. Integral operator representation. - The operator $\mathrm{R}$ can clearly be projected to an operator $\mathrm{R}_{s_{2} s_{1}} \equiv\left(\pi_{s_{2}} \otimes \pi_{s_{1}}\right) \mathrm{R}$ on $\mathscr{P}_{s_{2}} \otimes \mathscr{P}_{s_{1}}$. The action of this operator admits a representation by means of a distributional kernel:

Theorem 4. - Let $\tilde{\psi}\left(k_{2}, k_{1}\right)=\int_{\mathbb{R}} d x_{2} d x_{1} e^{2 \pi i\left(k_{1} x_{1}+k_{2} x_{2}\right)} \psi\left(x_{2}, x_{1}\right)$ be a Fourier transform of $\psi\left(x_{2}, x_{1}\right) \in \mathscr{P}_{s_{2}} \otimes \mathscr{P}_{s_{1}}$. The action of the R-operator on $\mathscr{P}_{s_{2}} \otimes \mathscr{P}_{s_{1}}$ admits the following representations as an integral operator in "coordinate" and "momentum" space respectively:

$$
\begin{aligned}
& \left(\mathrm{R}_{s_{2} s_{1}} \psi\right)\left(x_{2}, x_{1}\right)=\int_{\mathbb{R}} d x_{2}^{\prime} d x_{1}^{\prime} \mathrm{R}_{s_{2} s_{1}}\left(x_{2}, x_{1} \mid x_{2}^{\prime}, x_{1}^{\prime}\right) \psi\left(x_{2}^{\prime}, x_{1}^{\prime}\right), \\
& \left(\mathrm{R}_{s_{2} s_{1}} \tilde{\psi}\right)\left(k_{2}, k_{1}\right)=\int_{\mathbb{R}} d k_{2}^{\prime} d k_{1}^{\prime} \tilde{\mathrm{R}}_{s_{2} s_{1}}\left(k_{2}, k_{1} \mid k_{2}^{\prime}, k_{1}^{\prime}\right) \tilde{\psi}\left(k_{2}^{\prime}, k_{1}^{\prime}\right),
\end{aligned}
$$

with the kernels given by

$$
\begin{aligned}
& \mathrm{R}_{s_{2} s_{1}}\left(x_{2}, x_{1} \mid x_{2}^{\prime}, x_{1}^{\prime}\right)=e^{2 \pi i\left(s_{1}\left(x_{1}^{\prime}-x_{1}\right)+s_{2}\left(x_{2}-x_{2}^{\prime}\right)+\frac{i Q}{2}\left(x_{2}+x_{2}^{\prime}-x_{1}-x_{1}^{\prime}\right)+s_{1} s_{2}+\frac{1}{4} Q^{2}\right)} \\
& \quad \times \frac{G_{b}\left(\frac{Q}{2}+\frac{i}{2}\left(s_{1}+s_{2}\right)+i\left(x_{2}-x_{1}\right)\right)}{G_{b}\left(Q+\frac{i}{2}\left(s_{1}-s_{2}\right)+i\left(x_{2}-x_{1}^{\prime}\right)\right)} \frac{G_{b}\left(\frac{Q}{2}-\frac{i}{2}\left(s_{1}+s_{2}\right)+i\left(x_{2}^{\prime}-x_{1}^{\prime}\right)\right)}{G_{b}\left(Q+\frac{i}{2}\left(s_{2}-s_{1}\right)+i\left(x_{2}^{\prime}-x_{1}\right)\right)},
\end{aligned}
$$




$$
\begin{aligned}
& \tilde{\mathrm{R}}_{s_{2} s_{1}}\left(k_{2}, k_{1} \mid k_{2}^{\prime}, k_{1}^{\prime}\right)= \\
& \quad=\delta\left(k_{2}^{\prime}+k_{1}^{\prime}-k_{2}-k_{1}\right) \frac{e^{-\pi i\left(k_{1}^{\prime} k_{2}+k_{1} k_{2}^{\prime}\right)}}{G_{b}\left(Q+i\left(k_{1}^{\prime}-k_{1}\right)\right)} \frac{w_{b}\left(s_{1}+k_{1}\right)}{w_{b}\left(s_{1}+k_{1}^{\prime}\right)} \frac{w_{b}\left(s_{2}-k_{2}\right)}{w_{b}\left(s_{2}-k_{2}^{\prime}\right)} .
\end{aligned}
$$

The functions $w_{b}(x)$ and $G_{b}(x)$ are close relatives of the function $g_{b}(x)$ that will be defined in Subsection 3.1 below, and $G^{-1}(Q+i x)$ is taken as a short notation for the distribution $G^{-1}(Q+i(x+i 0))$.

2.9. Highest weight representations. - In order to demonstrate that the R-operator we are considering here indeed deserves to be called "universal" we are now going to show that the usual R-matrix for highest weight representations of $U_{q}(\mathfrak{s l}(2, \mathbb{R}))$ can be extracted from the analytic properties of the matrix elements given in Theorem 4 .

As a preparation let us consider the representation of $U_{q}(\mathfrak{s l}(2, \mathbb{R}))$ on the dual space $\mathscr{P}_{s}^{\prime}$ of $\mathscr{P}_{s}$. An interesting class of elements of $\mathscr{P}_{s}^{\prime}$ is furnished by the (complexified) delta-functionals $\delta_{k}$,

$$
\left\langle\boldsymbol{\delta}_{k}, f\right\rangle=f(k) .
$$

The $\delta_{k}$ are well-defined for all $k \in \mathbb{C} \backslash \mathscr{S}_{s}$, and the action of $U_{q}(\mathfrak{s l}(2, \mathbb{R}))$ is realized by

$$
\begin{aligned}
& \mathrm{E}_{s}^{t} \delta_{k}=+\left[\frac{Q}{2 b}-\frac{i}{b}(k-s)\right]_{q} \delta_{k+i b} \\
& \mathrm{~F}_{s}^{t} \delta_{k}=-\left[\frac{Q}{2 b}+\frac{i}{b}(k+s)\right]_{q} \delta_{k-i b}
\end{aligned} \quad \mathrm{~K}_{s}^{t} \delta_{k}=e^{-\pi b k} \delta_{k},
$$

where $[t]_{q} \equiv \frac{\sin \left(\pi b^{2} t\right)}{\sin \left(\pi b^{2}\right)}$ is the standard definition of a q-number, and the superscript " $t$ " on the generators indicates transposition.

Let us restrict attention to the set $\mathscr{D}_{s}$ of functionals $\delta_{k}$ for which $k$ is an element of $\left\{k=-s+i\left(\frac{Q}{2}+n b\right), n \in \mathbb{Z}^{\geq 0}\right\}$. It is easy to verify that (2.31) realizes a highest weight representation on $\mathscr{D}_{s}$.

Theorem 5. - The action of $\mathrm{R}^{t}$ on $\mathscr{H} \otimes \mathscr{D}_{s}$ is given by

$$
\mathrm{R}_{s}^{+}=q^{\mathrm{H} \otimes \mathrm{H}_{s}} \sum_{n=0}^{\infty} \frac{q^{\frac{1}{2}\left(n^{2}-n\right)}}{\prod_{k=1}^{n}[k]_{q}}\left(\left(q-q^{-1}\right) \mathrm{E} \otimes \mathrm{F}_{s}\right)^{n} q^{\mathrm{H} \otimes \mathrm{H}_{s}} .
$$

\subsection{Diagonalization of the R-operator. -}

Theorem 6. - The Clebsch-Gordan maps $\mathrm{C}\left[s_{3} \mid s_{2}, s_{1}\right]$ diagonalize the R-operator in the following sense:

$$
\mathrm{C}\left[s_{3} \mid s_{1}, s_{2}\right] \mathrm{B}_{s_{2} s_{1}}=\Omega\left(s_{3} \mid s_{2}, s_{1}\right) \mathrm{C}\left[s_{3} \mid s_{2}, s_{1}\right],
$$

with eigenvalue $\Omega\left(s_{3} \mid s_{2}, s_{1}\right)$ given as

$$
\Omega\left(s_{3} \mid s_{2}, s_{1}\right)=e^{-\pi i\left(h_{s_{3}}-h_{s_{2}}-h_{s_{1}}\right)}, \quad h_{s} \equiv s^{2}-\frac{Q^{2}}{4} .
$$


2.11. The strong coupling regime $|b|=1$. - We would finally like to point out that our results carry over to the strong coupling regime $b=e^{i \theta}, \theta \in[0, \pi / 2)$. This is almost obvious for those results whose proof relies mainly on the properties of the special functions $g_{b}(x), G_{b}(x)$ and $w_{b}(x)$. In this case the operators $\mathrm{E}, \mathrm{F}$ and $\mathrm{K}$ are normal (as follows from eqn. (3.19) below), and the hermitian conjugation acts as

$$
\mathrm{E}^{\dagger}=\tilde{\mathrm{E}}, \quad \mathrm{F}^{\dagger}=\tilde{\mathrm{F}}, \quad \mathrm{K}^{\dagger}=\tilde{\mathrm{K}} .
$$

Concerning the results that rely on [PT2] one may note that they all amount to certain identities between distributions that are defined by a standard analytic regularization in terms of the meromorphic functions $g_{b}(x), G_{b}(x)$ and $w_{b}(x)$. The relevant analytic properties underlying the validity of these identities all remain intact upon analytically continuing from the case of real $b$ to $|b|=1$.

\section{Preliminaries and auxiliary results}

3.1. Special functions. - The Double Gamma function $\Gamma_{2}\left(x \mid \omega_{1}, \omega_{2}\right)$ was introduced and studied by Barnes [Ba $]$. In what follows we will be dealing with (recall that $Q=b+b^{-1}$ )

$$
G_{b}(x) \equiv e^{\frac{\pi i}{2} x(x-Q)} \frac{\Gamma_{2}\left(x \mid b^{-1}, b\right)}{\Gamma_{2}\left(Q-x \mid b^{-1}, b\right)} .
$$

This function is closely related to the remarkable special functions introduced under the names of "quantum dilogarithm" in [FK1] and "quantum exponential function" in [W2]. $G_{b}(x)$ is a meromorphic function that has the following properties [Ba, $\mathbf{S h}$ :

$$
\begin{aligned}
\text { self-duality } & G_{b}(x)=G_{b^{-1}}(x), \\
\text { functional equation } & G_{b}(x+b)=\left(1-e^{2 \pi i b x}\right) G_{b}(x), \\
\text { reflection property } & G_{b}(x) G_{b}(Q-x)=e^{\pi i x(x-Q)}, \\
\text { complex conjugation } & \overline{G_{b}(x)=e^{\pi i \bar{x}(Q-\bar{x})} G_{b}(\bar{x}),} \\
\text { asymptotics } & G_{b}(x) \sim \begin{cases}\bar{\zeta}_{b} & \text { for } \mathfrak{I}(x) \rightarrow+\infty \\
\zeta_{b} e^{\pi i x(x-Q)} & \text { for } \mathfrak{I}(x) \rightarrow-\infty\end{cases} \\
G_{b}(x) \text { has poles at } & x=-n b-m b^{-1} \quad n, m \in \mathbb{Z}^{\geq 0}, \\
G_{b}(x) \text { has zeros at } & x=Q+n b+m b^{-1}
\end{aligned}
$$

where $\zeta_{b}=e^{\frac{\pi i}{4}+\frac{\pi i}{12}\left(b^{2}+b^{-2}\right)}$. By Proposition 5 in [Sh], the $G_{b}$-function admits for $\mathfrak{I} b^{2}>$ 0 the following infinite product representation

$$
G_{b}(x)=\bar{\zeta}_{b} \frac{\prod_{n=1}^{\infty}\left(1-e^{2 \pi i b^{-1}\left(x-n b^{-1}\right)}\right)}{\prod_{n=0}^{\infty}\left(1-e^{2 \pi i b(x+n b)}\right)} .
$$


We are also going to use two other functions that are closely related to $G_{b}(x)$, namely

$$
g_{b}(x) \equiv \frac{\bar{\zeta}_{b}}{G_{b}\left(\frac{Q}{2}+\frac{1}{2 \pi i b} \log x\right)}, \quad \text { and } \quad w_{b}(x) \equiv e^{\frac{\pi i}{2}\left(\frac{Q^{2}}{4}+x^{2}\right)} G_{b}\left(\frac{Q}{2}-i x\right) .
$$

The representation (2.22) for $g_{b}$ introduced above follows easily from the integral representation for the Double Gamma function introduced in [Sh].

For the reader's convenience we shall also list the relevant properties of $w_{b}(x)$ that follow from (3.2)- 3.7).

$$
\begin{aligned}
\text { self-duality } & w_{b}(x)=w_{b^{-1}}(x), \\
\text { functional equation } & w_{b}(x+i b)=2 w_{b}(x) \sin \pi b\left(\frac{Q}{2}-i x\right), \\
\text { reflection property } & w_{b}(x) w_{b}(-x)=1, \\
\text { complex conjugation } & \overline{w_{b}(x)}=w_{b}(-\bar{x}), \\
w_{b}(x) \text { has poles at } & x=-i\left(\frac{Q}{2}+n b+m b^{-1}\right) \quad \\
w_{b}(x) \text { has zeros at } & x=i\left(\frac{Q}{2}+n b+m b^{-1}\right) \quad n, m \in \mathbb{Z}^{\geq 0} .
\end{aligned}
$$

Notice that $\left|w_{b}(x)\right|=1$ if $x$ is real. Hence $w_{b}(\mathrm{X})$ is unitary if $\mathrm{X}$ is a self-adjoint operator.

\subsection{Operator algebraic preliminaries. -}

Lemma 3. - Let $A$ and $B$ be self-adjoint operators such that $[A, B]=2 \pi i$. Let $\varphi(t)$ be a function on the positive real axis and let $\gamma=\frac{1}{b^{2}}$. Then we have

$$
\begin{gathered}
\varphi(u+v)=w_{b}(2 \pi(A-B)) \varphi\left(e^{\frac{b}{2}(A+B)}\right) w_{b}(2 \pi(B-A)), \\
(u+v)^{\gamma}=u^{\gamma}+v^{\gamma},
\end{gathered}
$$

where $u=e^{b A}, v=e^{b B}$.

Proof. - It is convenient to introduce $p \equiv \frac{B-A}{2 \pi}$ and $x \equiv \frac{A+B}{4 \pi}$. Observe that $[p, x]=\frac{1}{2 \pi i}$; so that we have

$$
f(p) e^{\pi b x}=e^{\pi b x} f\left(p-i \frac{b}{2}\right) .
$$

for any function $f(t)$ that is bounded and analytic in the strip $\frac{b}{2} \leq \mathfrak{I}(p) \leq 0$. Using the Baker-Campbell-Hausdorff formula for Weyl-type operators and the properties of the function $w_{b}$, we may calculate as follows:

$$
\begin{aligned}
& w_{b}(-p) e^{2 \pi b x} w_{b}(p) \stackrel{\text { 3.12) }}{=} \frac{1}{w_{b}(p)} e^{2 \pi b x} w_{b}(p) \stackrel{\text { 3.17) }}{=} e^{\pi b x} \frac{w_{b}\left(p+i \frac{b}{2}\right)}{w_{b}\left(p-i \frac{b}{2}\right)} e^{\pi b x} \\
& \stackrel{3.11)}{=} 2 e^{\pi b x}(\cosh \pi b p) e^{\pi b x}=e^{\frac{b}{4}(A+B)}\left(e^{\frac{b}{2}(B-A)}+e^{\frac{b}{2}(A-B)}\right) e^{\frac{b}{4}(A+B)} \\
&=u+v
\end{aligned}
$$

The last expression is therefore unitarily equivalent to the positive self-adjoint operator $e^{2 \pi b x}$. Our claim (3.15) follows by applying the standard functional calculus of selfadjoint operators. 
Relation (3.16) can be proven along the same lines taking into account that, thanks to self-duality (3.10), $w_{b}$ obeys also the equation

$$
w_{b}\left(x+\frac{i}{b}\right)=2 w_{b}(x) \sin \left(\frac{\pi}{b}\left(\frac{Q}{2}-i x\right)\right) .
$$

Therefore, for $\varphi(t)=t^{\gamma}$ we have

$$
\begin{aligned}
(u+v)^{\gamma} & =w_{b}(-p) e^{\frac{2 \pi}{b} x} w_{b}(p) \stackrel{3.17}{=} e^{\frac{\pi}{b} x} \frac{w_{b}\left(p+\frac{i}{2 b}\right)}{w_{b}\left(p-\frac{i}{2 b}\right)} e^{\frac{\pi}{b} x} \\
& \stackrel{3.18}{=} 2 e^{\frac{\pi}{b} x}\left(\cosh \frac{\pi}{b} p\right) e^{\frac{\pi}{b} x}=e^{\frac{1}{4 b}(A+B)}\left(e^{\frac{1}{2 b}(B-A)}+e^{\frac{1}{2 b}(A-B)}\right) e^{\frac{1}{4 b}(A+B)}=u^{\gamma}+v^{\gamma} .
\end{aligned}
$$

Remark 3. - Another way to prove relation (3.16) in Lemma 3 is to use the $b$ binomial formula (B.4) that we derive in Appendix. When $t$ approaches the value $-i \gamma$, the $b$-binomial coefficient (B.5) vanishes unless $\tau$ takes special values determined by (3.7). Furthermore, for $t=-i \gamma$ the $b$-binomial coefficient has nonvanishing residues only at $\tau=0$ and $\tau=-i \gamma$. The contributions from these two poles yield the two terms on the r.h.s. of (3.16). Similar consideration for $t$ approaching $-i n \gamma, n>1$ shows that the $b$-binomial coefficient has nonvanishing residues at $\tau=0,-i \gamma, \ldots,-i n \gamma$. Therefore $(u+v)^{n \gamma}$ can be represented as sum of $(n+1)$ terms which is analogous to the $q$-binomial formula in the compact case.

The proven Lemma leads to useful representations for the generators and the R-operator of $U_{q}(\operatorname{sl}(2, \mathbb{R}))$. For brevity, we denote $\mathrm{e}_{b} \equiv\left(2 \sin \pi b^{2}\right) \mathrm{E}$ and $\mathrm{f}_{b} \equiv$ $\left(2 \sin \pi b^{2}\right) \mathrm{F}$, whereas $e_{\frac{1}{b}}$ and $\mathrm{f}_{\frac{1}{b}}$ will stand for their counterparts with $b$ replaced by $\frac{1}{b}$.

Lemma 4. $-\pi_{\mathscr{H}}(E)$ and $\pi_{\mathscr{H}}(F)$ admit the following representation:

$$
\mathrm{e}_{b}=w_{b}\left(\mathrm{p}_{2}\right) e^{2 \pi b\left(\mathrm{x}_{1}-\mathrm{x}_{2}\right)} w_{b}\left(-\mathrm{p}_{2}\right), \quad \mathrm{f}_{b}=w_{b}\left(\mathrm{p}_{1}\right) e^{2 \pi b\left(\mathrm{x}_{2}-\mathrm{x}_{1}\right)} w_{b}\left(-\mathrm{p}_{1}\right) .
$$

$\mathrm{R}$ may be represented as follows:

$$
\begin{aligned}
\mathrm{R}=q^{\mathrm{H} \otimes \mathrm{H}}\left(w_{b}\left(\mathrm{p}_{2}\right) \otimes w_{b}\left(\mathrm{p}_{1}\right)\right) g_{b}\left(e^{2 \pi b\left(\mathrm{x}_{1}-\mathrm{x}_{2}\right)} \otimes e^{2 \pi b\left(\mathrm{x}_{2}-\mathrm{x}_{1}\right)}\right) \\
\cdot\left(w_{b}\left(-\mathrm{p}_{2}\right) \otimes w_{b}\left(-\mathrm{p}_{1}\right)\right) q^{\mathrm{H} \otimes \mathrm{H}} .
\end{aligned}
$$

Proof. - In Lemma 3, we can identify $\frac{1}{2 \pi}(A-B)=\mathrm{p}_{n+1}$ and $\frac{1}{4 \pi}(A+B)=\mathrm{x}_{n}-\mathrm{x}_{n+1}$, where $n=1,2$ (with convention that $n+2 \equiv n$ ). Then, as seen from the definition (2.5), we have $u+v=\mathrm{e}_{b}$ for $n=1$ and $u+v=\mathrm{f}_{b}$ for $n=2$. Therefore, (3.19) is just a particular case of (3.15). Furthermore, for functions $\varphi(t)$ defined on $\mathbb{R}^{+}$we have

$$
\varphi\left(\mathrm{e}_{b}\right)=w_{b}\left(\mathrm{p}_{2}\right) \varphi\left(e^{2 \pi b\left(\mathrm{x}_{1}-\mathrm{x}_{2}\right)}\right) w_{b}\left(-\mathrm{p}_{2}\right), \quad \varphi\left(\mathrm{f}_{b}\right)=w_{b}\left(\mathrm{p}_{1}\right) \varphi\left(e^{2 \pi b\left(\mathrm{x}_{2}-\mathrm{x}_{1}\right)}\right) w_{b}\left(-\mathrm{p}_{1}\right) .
$$

In particular, we can take $\varphi(t)=g_{b}(x)$ (recall that $\left|g_{b}(x)\right|=1$ for $x \in \mathbb{R}^{+}$). For this choice of $\varphi(t)$, the representation (3.20) for $\mathrm{R}$ follows immediately from the definition (2.23). 
Corollary 1. - For $\gamma=\frac{1}{b^{2}}$ we have

$$
\left(\mathrm{e}_{b}\right)^{\gamma}=\mathrm{e}_{\frac{1}{b}}, \quad\left(\mathrm{f}_{b}\right)^{\gamma}=\mathrm{f}_{\frac{1}{b}} .
$$

Proof. - Notice that, if $u$ and $v$ are identified as in the proof of Lemma 4 , then $u^{\gamma}+v^{\gamma}=\mathrm{e}_{\frac{1}{5}}$ for $n=1$ and $u^{\gamma}+v^{\gamma}=\mathrm{f}_{\frac{1}{5}}$ for $n=2$. Thus, relations (3.22) are a particular case of (3.16) .

This proves the relations (2.9) from Lemma 1.

Corollary 2. - The definition of the R-operator proposed in [[F2],

$$
\mathrm{R}=q^{\mathrm{H} \otimes \mathrm{H}} \frac{\prod_{n=0}^{\infty}\left(1+q^{2 n+1} \mathrm{e}_{b} \otimes \mathrm{f}_{b}\right)}{\prod_{n=0}^{\infty}\left(1+\tilde{q}^{2 n+1} \mathrm{e}_{\frac{1}{b}} \otimes \mathrm{f}_{\frac{1}{b}}\right)} q^{\mathrm{H} \otimes \mathrm{H}}, \quad \tilde{q}=e^{-i \pi b^{-2}},
$$

which is valid for $b=e^{i \vartheta}, \vartheta \in\left(0, \frac{\pi}{2}\right)$, coincides with our definition (2.23).

Proof. -

$$
\begin{aligned}
& q^{-\mathrm{H} \otimes \mathrm{H}} \mathrm{R} q^{-\mathrm{H} \otimes \mathrm{H} \stackrel{(2.23)}{=}} g_{b}\left(\mathrm{e}_{b} \otimes \mathrm{f}_{b}\right) \stackrel{(2.22)}{=} \bar{\zeta}_{b}\left(G_{b}\left(\frac{Q}{2}+\frac{1}{2 \pi i b} \log \left(\mathrm{e}_{b} \otimes \mathrm{f}_{b}\right)\right)\right)^{-1} \\
& \stackrel{\text { (3.8) }}{=} \frac{\prod_{n=0}^{\infty}\left(1-e^{2 \pi i b\left(\frac{Q}{2}+n b\right)} \mathrm{e}_{b} \otimes \mathrm{f}_{b}\right)}{\prod_{n=1}^{\infty}\left(1-e^{2 \pi i b^{-1}\left(\frac{Q}{2}-n b^{-1}\right)}\left(\mathrm{e}_{b} \otimes \mathrm{f}_{b}\right)^{\frac{1}{b^{2}}}\right.} \stackrel{\text { 3.22 }}{=} \frac{\prod_{n=0}^{\infty}\left(1+q^{2 n+1} \mathrm{e}_{b} \otimes \mathrm{f}_{b}\right)}{\prod_{n=0}^{\infty}\left(1+\tilde{q}^{2 n+1} \mathrm{e}_{\frac{1}{b}} \otimes \mathrm{f}_{\frac{1}{b}}\right)} .
\end{aligned}
$$

Corollary 3. $-\pi_{\mathscr{H}}(E)$ and $\pi_{\mathscr{H}}(F)$ admit the following representation:

$$
\mathrm{e}_{b}=e^{2 \pi b\left(\mathrm{x}_{1}-\mathrm{x}_{2}\right)+b \psi_{b}\left(\mathrm{p}_{2}\right)}, \quad \mathrm{f}_{b}=e^{2 \pi b\left(\mathrm{x}_{2}-\mathrm{x}_{1}\right)+b \psi_{b}\left(\mathrm{p}_{1}\right)}
$$

where $\psi_{b}(t) \equiv i \partial_{t}\left(\log w_{b}(t)\right)$.

Proof. - Eqs. (3.21) for $\varphi(t)=\log (t)$ yield

$$
\begin{aligned}
\log \left(\mathrm{e}_{b}\right) & =w_{b}\left(\mathrm{p}_{2}\right) 2 \pi b\left(\mathrm{x}_{\mathbf{1}}-\mathrm{x}_{2}\right) w_{b}\left(-\mathrm{p}_{2}\right)=2 \pi b\left(\mathrm{x}_{\mathbf{1}}-\mathrm{x}_{2}\right) \\
& +2 \pi b\left[w_{b}\left(\mathrm{p}_{2}\right),\left(\mathrm{x}_{\mathbf{1}}-\mathrm{x}_{2}\right)\right] w_{b}\left(-\mathrm{p}_{2}\right)=2 \pi b\left(\mathrm{x}_{\mathbf{1}}-\mathrm{x}_{2}\right)+\left.i b \partial_{t} w_{b}(t)\right|_{t=\mathrm{p}_{2}} \frac{1}{w_{b}\left(\mathrm{p}_{2}\right)}
\end{aligned}
$$

and, analogously, $\log \left(\mathrm{f}_{b}\right)=2 \pi b\left(\mathrm{x}_{2}-\mathrm{x}_{1}\right)+b \psi_{b}\left(\mathrm{p}_{1}\right)$. Exponentiating these relations, we obtain (3.24).

Remark 4. - Alternatively, eqs. (3.24) can be derived from (2.5) with the help of the Baker-Campbell-Hausdorff formula. Observe also that $\left(\log \mathrm{e}_{b}+\log \mathrm{f}_{b}\right)=b\left(\psi_{b}\left(\mathrm{p}_{1}\right)+\right.$ $\left.\psi_{b}\left(\mathrm{p}_{2}\right)\right)$ commutes with $\mathrm{C}$ and $\mathrm{H}$.

We may now give the proof of Lemma 2. In Lemma 3, let us choose $A=\left(2 \pi\left(\mathrm{x}_{1}-\right.\right.$ $\left.\left.\mathrm{x}_{2}\right)+\psi_{b}\left(\mathrm{p}_{2}\right)\right) \otimes 1+1 \otimes \frac{\pi}{2}\left(\mathrm{p}_{2}-\mathrm{p}_{1}\right)$ and $B=1 \otimes\left(2 \pi\left(\mathrm{x}_{1}-\mathrm{x}_{2}\right)+\psi_{b}\left(\mathrm{p}_{2}\right)\right)+\frac{\pi}{2}\left(\mathrm{p}_{1}-\mathrm{p}_{2}\right) \otimes$ 
1. In view of (3.24) this implies the identification $u=\mathrm{e}_{b} \otimes \mathrm{K}_{b}$ and $v=\mathrm{K}_{b}^{-1} \otimes \mathrm{e}_{b}$. Therefore, we have

$$
\begin{aligned}
\Delta\left(\mathrm{e}_{\frac{1}{b}}\right) & \stackrel{3.22}{=} \Delta\left(\left(\mathrm{e}_{b}\right)^{\frac{1}{b^{2}}}\right)=\left(\Delta\left(\mathrm{e}_{b}\right)\right)^{\frac{1}{b^{2}}} \stackrel{(2.11)}{=}\left(\mathrm{e}_{b} \otimes \mathrm{K}_{b}+\mathrm{K}_{b}^{-1} \otimes \mathrm{e}_{b}\right)^{\frac{1}{b^{2}}} \\
& \stackrel{3.16}{=}\left(\mathrm{e}_{b} \otimes \mathrm{K}_{b}\right)^{\frac{1}{b^{2}}}+\left(\mathrm{K}_{b}^{-1} \otimes \mathrm{e}_{b}\right)^{\frac{1}{b^{2}}} \stackrel{(3.22)}{=} \mathrm{e}_{\frac{1}{b}} \otimes \mathrm{K}_{\frac{1}{b}}+\mathrm{K}_{\frac{1}{b}}^{-1} \otimes \mathrm{e}_{\frac{1}{b}} .
\end{aligned}
$$

The relation for $\tilde{\mathrm{F}}$ in (2.13) is proven similarly.

Lemma 5. - Let the powers $E^{\alpha}$ and $F^{\alpha}$ with $\mathfrak{I} \alpha \neq 0$ be defined on $\mathscr{H}$ in the sense of (3.21). Then we have

$$
\left[\mathrm{E}, \mathrm{F}^{\alpha}\right]=[\alpha]_{q}[2 \mathrm{H}+\alpha-1]_{q} \mathrm{~F}^{\alpha-1}, \quad\left[\mathrm{E}^{\alpha}, \mathrm{F}\right]=[\alpha]_{q}[2 \mathrm{H}-\alpha+1]_{q} \mathrm{E}^{\alpha-1},
$$

where the q-numbers are defined as in Theorem 5 .

Proof. -

$$
\begin{aligned}
& {\left[\mathrm{E}, \mathrm{F}^{\alpha}\right] \stackrel{\text { 3.19) }}{=}\left[w_{b}\left(\mathrm{p}_{2}\right) \frac{e^{2 \pi b\left(\mathrm{x}_{1}-\mathrm{x}_{2}\right)}}{2 \sin \pi b^{2}} w_{b}\left(-\mathrm{p}_{2}\right), w_{b}\left(\mathrm{p}_{1}\right) \frac{e^{2 \pi \alpha b\left(\mathrm{x}_{2}-\mathrm{x}_{1}\right)}}{\left(2 \sin \pi b^{2}\right)^{\alpha}} w_{b}\left(-\mathrm{p}_{1}\right)\right]} \\
& \stackrel{\text { 3.17) }}{=}\left(\frac{w_{b}\left(\mathrm{p}_{1}+i b\right) w_{b}\left(\mathrm{p}_{2}\right)}{w_{b}\left(\mathrm{p}_{1}\right) w_{b}\left(\mathrm{p}_{2}-i b\right)}-\frac{w_{b}\left(\mathrm{p}_{1}+(1-\alpha) i b\right) w_{b}\left(\mathrm{p}_{2}+\alpha i b\right)}{w_{b}\left(\mathrm{p}_{1}-\alpha i b\right) w_{b}\left(\mathrm{p}_{2}+(\alpha-1) i b\right)}\right) \\
& \times w_{b}\left(\mathrm{p}_{1}\right) \frac{e^{2 \pi(\alpha-1) b\left(\mathrm{x}_{2}-\mathrm{x}_{1}\right)}}{\left(2 \sin \pi b^{2}\right)^{\alpha+1}} w_{b}\left(-\mathrm{p}_{1}\right) \\
& \quad\left(\left[\frac{Q}{2 b}-\frac{i \mathrm{p}_{1}}{b}\right]_{q}\left[\frac{Q}{2 b}+\frac{i \mathrm{p}_{2}}{b}\right]_{q}-\left[\frac{Q}{2 b}-\alpha-\frac{i \mathrm{p}_{1}}{b}\right]_{q}\left[\frac{Q}{2 b}-\alpha+\frac{i \mathrm{p}_{2}}{b}\right]_{q}\right) \mathrm{F}^{\alpha-1} \\
& =[\alpha]_{q}\left[\frac{Q}{b}-\alpha+\frac{i}{b}\left(\mathrm{p}_{2}-\mathrm{p}_{1}\right)\right]_{q} \mathrm{~F}^{\alpha-1}=[\alpha]_{q}\left[\frac{1}{i b}\left(\mathrm{p}_{2}-\mathrm{p}_{1}\right)+\alpha-1\right]_{q} \mathrm{~F}^{\alpha-1} \\
& \quad 2.5]_{q}[2 \mathrm{H}+\alpha-1]_{q} \mathrm{~F}^{\alpha-1} .
\end{aligned}
$$

In the fourth line we used the definition of $q$-number; in the fifth line we used the identities $[x]_{q}[y]_{q}-[x-\alpha]_{q}[y-\alpha]_{q}=[\alpha]_{q}[x+y-\alpha]_{q}$ and $\left[t+b^{-2}\right]_{q}=-[t]_{q}$. The second formula in (3.25) is derived analogously.

Lemma 6. - Let $A$ and $B$ be self-adjoint operators such that $[A, B]=2 \pi i$. Then for the function $g_{b}(x)$ defined in (2.22) we have

$$
\begin{aligned}
& g_{b}(u) g_{b}(v)=g_{b}(u+v), \\
& g_{b}(v) g_{b}(u)=g_{b}(u) g_{b}\left(q^{-1} u v\right) g_{b}(v),
\end{aligned}
$$

where $u=e^{b A}, v=e^{b B}$ and $q=e^{i \pi b^{2}}$. Furthermore, (3.26) $\Leftrightarrow$ (3.27).

In the literature, eqs. (3.26) and (3.27) are often referred to as the quantum exponential and the quantum pentagon relations. They also hold for the function $s_{q}(x)=\prod_{n=0}^{\infty}\left(1+x q^{2 n+1}\right)$ which is the compact counterpart of $g_{b}(x)$. For $s_{q}(x)$, the quantum exponential relation has been known since long time $[\mathbf{S c h}]$ and the quantum pentagon relation was found in [[FV]. 
Since (3.26) and (3.27) are equivalent, it suffices to prove one of them. Proofs of the quantum pentagon relation were given in [ $\mathbf{F K V}]$ and [W2]. Nevertheless, we find it instructive to give another proof of the quantum exponential relation in Appendix B since it will allow us to introduce the notion of $b$-binomial coefficients.

\subsection{Alternative representations of the R-operator. -}

Lemma 7. - $\mathrm{R}$ and $\mathrm{R}^{-1}$ may be decomposed into powers of $\mathrm{E} \otimes \mathrm{F}$ as follows:

$$
\begin{aligned}
\mathrm{R} & =b \int_{\mathbb{R}} d t \frac{e^{-\pi i b^{2} t^{2}}}{G_{b}(Q+i b t)} q^{\mathrm{H} \otimes \mathrm{H}}\left(4\left(\sin \pi b^{2}\right)^{2} \mathrm{E} \otimes \mathrm{F}\right)^{i t} q^{\mathrm{H} \otimes \mathrm{H}}, \\
\mathrm{R}^{-1} & =b \int_{\mathbb{R}} d t \frac{e^{-\pi b Q t}}{G_{b}(Q+i b t)} q^{-\mathrm{H} \otimes \mathrm{H}}\left(4\left(\sin \pi b^{2}\right)^{2} \mathrm{E} \otimes \mathrm{F}\right)^{i t} q^{-\mathrm{H} \otimes \mathrm{H}},
\end{aligned}
$$

where the integration contour goes above the pole at $t=0$.

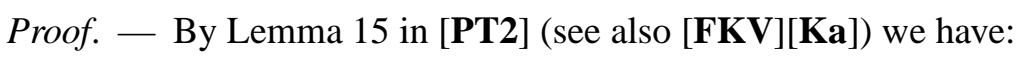

$$
\int_{\mathbb{R}} d \tau e^{-2 \pi \tau \beta} \frac{G_{b}(\alpha+i \tau)}{G_{b}(Q+i \tau)}=\frac{G_{b}(\alpha) G_{b}(\beta)}{G_{b}(\alpha+\beta)} .
$$

The function $\frac{1}{G_{b}(Q+i \tau)}$ has a pole at $\tau=0$ and is analytic in the upper half-plane. The integration contour in (3.30) goes above this pole.

Considering the asymptotics of (3.30) for $\mathfrak{I} \alpha \rightarrow-\infty$ and $\mathfrak{I} \alpha \rightarrow+\infty$, using the properties (3.4) and (3.6), and making a change of variables, we obtain the following Fourier transformation formulae

$$
\begin{aligned}
& b \int_{\mathbb{R}+i 0} d t e^{2 \pi i b t r} \frac{e^{-\pi i b^{2} t^{2}}}{G_{b}(Q+i b t)}=\frac{\bar{\zeta}_{b}}{G_{b}\left(\frac{Q}{2}-i r\right)}=g_{b}\left(e^{2 \pi b r}\right), \\
& b \int_{\mathbb{R}+i 0} d t e^{2 \pi i b t r} \frac{e^{-\pi b Q t}}{G_{b}(Q+i b t)}=\zeta_{b} G_{b}\left(\frac{Q}{2}-i r\right)=\left(g_{b}\left(e^{2 \pi b r}\right)\right)^{-1} .
\end{aligned}
$$

Lemma 7 follows if we put here $r=\frac{1}{2 \pi b} \log \left(4\left(\sin \pi b^{2}\right)^{2} \mathrm{E} \otimes \mathrm{F}\right)$ and compare the result with the definition (2.23).

We now come to the proof of Theorem 4 . Consider the product representation of the R-operator of Lemma $\bigoplus$ projected to $\mathscr{P}_{s_{2}} \otimes \mathscr{P}_{s_{1}}$ by means of the reduction described in Subsection 2.3,

$$
\begin{aligned}
\mathrm{R}_{s_{2} s_{1}} \stackrel{(3.20)}{=} q^{\mathrm{H}_{2}} & \otimes \mathrm{H}_{1}\left(w_{b}\left(s_{2}-\mathrm{p}\right) \otimes w_{b}\left(s_{1}+\mathrm{p}\right)\right) g_{b}\left(e^{2 \pi b \times} \otimes e^{-2 \pi b \times}\right) \\
& \times\left(w_{b}\left(s_{2}-\mathrm{p}\right) \otimes w_{b}\left(s_{1}+\mathrm{p}\right)\right)^{-1} q^{\mathrm{H}_{2} \otimes \mathrm{H}_{1}} \\
\stackrel{3.31}{=} q^{\mathrm{H}_{2} \otimes \mathrm{H}_{1}}\left(w_{b}\left(s_{2}-\mathrm{p}\right) \otimes w_{b}\left(s_{1}+\mathrm{p}\right)\right) \int_{\mathbb{R}} d \tau \frac{e^{-\pi i \tau^{2}} e^{2 \pi i \tau \times} \otimes e^{-2 \pi i \tau \times}}{G_{b}(Q+i \tau)} & \\
& \times\left(w_{b}\left(s_{2}-\mathrm{p}\right) \otimes w_{b}\left(s_{1}+\mathrm{p}\right)\right)^{-1} q^{\mathrm{H}_{2} \otimes \mathrm{H}_{1}}
\end{aligned}
$$


It is now easy to compute the "matrix elements" of $\mathrm{R}_{s_{2} s_{1}}$ on the states $\left|k_{2}, k_{1}\right\rangle=\left|k_{2}\right\rangle \otimes$ $\left|k_{1}\right\rangle$, where $|k\rangle \equiv e^{2 \pi i x k}$. Taking into account that $\mathrm{p}|k\rangle=k|k\rangle$ and $\left\langle k \mid k^{\prime}\right\rangle=\delta\left(k^{\prime}-k\right)$, we find

$$
\begin{aligned}
& \tilde{\mathrm{R}}_{s_{2} s_{1}}\left(k_{2}, k_{1} \mid k_{2}^{\prime}, k_{1}^{\prime}\right)=\left\langle k_{2}, k_{1}\left|\mathrm{R}_{s_{2} s_{1}}\right| k_{2}^{\prime}, k_{1}^{\prime}\right\rangle \\
& =\int_{\mathbb{R}} d \tau \frac{e^{-\pi i\left(\tau^{2}+k_{1} k_{2}+k_{1}^{\prime} k_{2}^{\prime}\right)}}{G_{b}(Q+i \tau)} \frac{w_{b}\left(s_{1}+k_{1}\right)}{w_{b}\left(s_{1}+k_{1}^{\prime}\right)} \frac{w_{b}\left(s_{2}-k_{2}\right)}{w_{b}\left(s_{2}-k_{2}^{\prime}\right)} \delta\left(k_{2}^{\prime}-k_{2}+\tau\right) \delta\left(k_{1}^{\prime}-k_{1}-\tau\right)
\end{aligned}
$$

which gives us the kernel (2.30) of the "momentum" representation in Theorem $\sharp$.

The kernel of the "coordinate" representation (2.27) can be obtained as a Fourier transform of (3.34):

$$
\begin{aligned}
\mathrm{R}_{s_{2} s_{1}}\left(x_{2},\right. & \left.x_{1} \mid x_{2}^{\prime}, x_{1}^{\prime}\right)=\int_{\mathbb{R}} d k_{2}^{\prime} d k_{1}^{\prime} d k_{2} d k_{1} e^{2 \pi i\left(x_{2} k_{2}+x_{1} k_{1}-x_{2}^{\prime} k_{2}^{\prime}-x_{1}^{\prime} k_{1}^{\prime}\right)} \tilde{\mathrm{R}}_{s_{2} s_{1}}\left(k_{2}, k_{1} \mid k_{2}^{\prime}, k_{1}^{\prime}\right) \\
= & \int_{\mathbb{R}} d \tau d k_{2}^{\prime} d k_{1}^{\prime} \frac{e^{\pi i\left(\tau\left(k_{2}^{\prime}-k_{1}^{\prime}\right)-2 k_{1}^{\prime} k_{2}^{\prime}\right)}}{G_{b}(Q+i \tau)} e^{2 \pi i\left(\tau\left(x_{2}-x_{1}\right)+k_{1}^{\prime}\left(x_{1}-x_{1}^{\prime}\right)+k_{2}^{\prime}\left(x_{2}-x_{2}^{\prime}\right)\right)} \\
& \times \frac{w_{b}\left(s_{1}+k_{1}^{\prime}-\tau\right)}{w_{b}\left(s_{1}+k_{1}^{\prime}\right)} \frac{w_{b}\left(s_{2}-k_{2}^{\prime}-\tau\right)}{w_{b}\left(s_{2}-k_{2}^{\prime}\right)}
\end{aligned}
$$

The remaining integrations are performed by using relation (3.30) three times. The result of this straightforward but tedious calculation is given by (2.29).

\section{Proofs of the main results}

4.1. Proof of Theorem 1: Co-associativity. - First, it is straightforward to write out $(\mathrm{id} \otimes \Delta) \circ \Delta(\mathrm{X})$ and $(\Delta \otimes \mathrm{id}) \circ \Delta(\mathrm{X})$ in terms of the Clebsch-Gordan maps $\mathrm{C}\left[s_{3} \mid s_{2}, s_{1}\right]$ :

$$
\begin{aligned}
& \left(\pi_{s_{3}} \otimes \pi_{s_{2}} \otimes \pi_{s_{1}}\right) \circ(\mathrm{id} \otimes \Delta) \circ \Delta(\mathrm{X})=\int_{\mathbb{R}^{+}} d \mathrm{~m}\left(s_{4}\right) d \mathrm{~m}\left(s_{21}\right) \mathrm{C}_{3(21)}^{\dagger}\left(s_{21}\right) \cdot \mathrm{X} \cdot \mathrm{C}_{3(21)}\left(s_{21}\right) \\
& \left(\pi_{s_{3}} \otimes \pi_{s_{2}} \otimes \pi_{s_{1}}\right) \circ(\Delta \otimes \mathrm{id}) \circ \Delta(\mathrm{X})=\int_{\mathbb{R}^{+}} d \mathrm{~m}\left(s_{4}\right) d \mathrm{~m}\left(s_{32}\right) \mathrm{C}_{(32) 1}^{\dagger}\left(s_{32}\right) \cdot \mathrm{X} \cdot \mathrm{C}_{(32) 1}\left(s_{32}\right),
\end{aligned}
$$

where we have introduced

$$
\begin{aligned}
& \mathrm{C}_{3(21)}\left(s_{21}\right) \equiv \mathrm{C}\left[s_{4} \mid s_{3}, s_{21}\right] \cdot\left(\mathrm{id} \otimes \mathrm{C}\left[s_{21} \mid s_{2}, s_{1}\right]\right), \\
& \mathrm{C}_{(32) 1}\left(s_{32}\right) \equiv \mathrm{C}\left[s_{4} \mid s_{32}, s_{1}\right] \cdot\left(\mathrm{C}\left[s_{32} \mid s_{3}, s_{2}\right] \otimes \mathrm{id}\right) .
\end{aligned}
$$

Proposition 7 in [PT2] is equivalent to

$$
\mathrm{C}_{3(21)}\left(s_{21}\right)=\int_{\mathbb{R}^{+}} d s_{32}\left\{\begin{array}{lll}
s_{1} & s_{2} & s_{21} \\
s_{3} & s_{4} & s_{32}
\end{array}\right\} \mathrm{C}_{(32) 1}\left(s_{32}\right),
$$


where $\left\{\begin{array}{lll}s_{1} & s_{2} & s_{21} \\ s_{3} & s_{4} & s_{32}\end{array}\right\}_{b}$ are the b-Racah-Wigner coefficients introduced in [PT2]. It follows that

$$
\begin{aligned}
& \left(\pi_{s_{3}} \otimes \pi_{s_{2}} \otimes \pi_{s_{1}}\right) \circ(\mathrm{id} \otimes \Delta) \circ \Delta(\mathrm{X})= \\
& =\int_{\mathbb{R}^{+}} d \mathrm{~m}\left(s_{4}\right) d \mathrm{~m}\left(s_{21}\right) \int_{\mathbb{R}^{+}} d \mathrm{~m}\left(s_{32}\right) d \mathrm{~m}\left(s_{32}^{\prime}\right)\left\{\begin{array}{lll}
s_{1} & s_{2} & s_{21} \\
s_{3} & s_{4} & s_{32}^{\prime}
\end{array}\right\}_{b}^{*}\left\{\begin{array}{lll}
s_{1} & s_{2} & s_{21} \\
s_{3} & s_{4} & s_{32}
\end{array}\right\}_{b} . \\
& \cdot \mathrm{C}_{(32) 1}^{\dagger}\left(s_{32}\right) \cdot \mathrm{X} \cdot \mathrm{C}_{(32) 1}\left(s_{32}\right) \text {. }
\end{aligned}
$$

Exchanging the integrations over $s_{21}$ and $s_{32}, s_{32}^{\prime}$, and using formula (89) from [PT2],

$$
\int_{\mathbb{R}^{+}} d \mathrm{~m}\left(s_{21}\right)\left\{\begin{array}{lll}
s_{1} & s_{2} & s_{21} \\
s_{3} & s_{4} & s_{32}^{\prime}
\end{array}\right\}_{b}^{*}\left\{\begin{array}{llll}
s_{1} & s_{2} & s_{21} \\
s_{3} & s_{4} & s_{32}
\end{array}\right\}_{b}=\mathrm{m}\left(s_{32}\right) \boldsymbol{\delta}\left(s_{32}-s_{32}^{\prime}\right),
$$

yields the claim.

4.2. Proof of Theorem 2: Invariance of the Haar-measure. - We shall consider the left invariant Haar measure $h_{1}$ only, the proof for the case of $h_{r}$ being completely analogous.

A few preparations are in order. The elements of $\mathscr{A}_{\mathrm{h}}^{1}$ can be represented as integral operators: If a vector $\psi \in \mathscr{H}$ is realized by a function $\psi(k, s)$, then

$$
(\mathrm{O} \psi)(k, s)=\int_{\mathbb{R}} d k^{\prime} K_{\mathrm{O}}\left(k, k^{\prime} \mid s\right) \psi\left(k^{\prime}, s\right) .
$$

In terms of the kernel $K_{\mathrm{O}}\left(k, k^{\prime} \mid s\right)$ one may write the defintion of $\mathrm{h}_{1}$ as

$$
\mathrm{h}_{1}(\mathrm{O})=\int_{\mathbb{R}^{+}} d \mathrm{~m}(s) \int_{\mathbb{R}} d k e^{-2 \pi Q k} K_{\mathrm{O}}(k, k \mid s) .
$$

The distributional matrix elements of an operator $\mathrm{O} \in \mathscr{A}$ are always of the form

$$
\left\langle s, k|\mathrm{O}| s^{\prime}, k^{\prime}\right\rangle \equiv \delta\left(s-s^{\prime}\right)\left\langle\left\langle k|\mathrm{O}| k^{\prime}\right\rangle\right\rangle_{s} .
$$

By using an analogous notation for operators in $\mathscr{A} \otimes \mathscr{A}$ one may represent the distributional matrix elements of $\Delta(\mathrm{O})$ as

$$
\begin{aligned}
& \left\langle\left\langle k_{2}, k_{1}|\Delta(\mathrm{O})| k_{2}^{\prime}, k_{1}^{\prime}\right\rangle\right\rangle_{s_{2} s_{1}} \\
& \left.\quad=\int_{\mathbb{R}^{+}} d \mathrm{~m}\left(\mathrm{~s}_{3}\right) \int_{\mathbb{R}} d k_{3} d k_{3}^{\prime} K_{\mathrm{O}}\left(k_{3}, k_{3}^{\prime} \mid s_{3}\right)\left(\begin{array}{ccc}
s_{3} & s_{2} & s_{1} \\
k_{3} & k_{2} & k_{1}
\end{array}\right]\right)^{*}\left[\begin{array}{ccc}
s_{3} & s_{2} & s_{1} \\
k_{3}^{\prime} & k_{2}^{\prime} & k_{1}^{\prime}
\end{array}\right] .
\end{aligned}
$$

In order to make the justification for the following manipulations more transparent, we are going to employ the following regularization for the distributions involved:

$$
\left[\begin{array}{lll}
s_{3} & s_{2} & s_{1} \\
k_{3} & k_{2} & k_{1}
\end{array}\right]=\lim _{\varepsilon \downarrow 0}\left[\begin{array}{lll}
s_{3} & s_{2} & s_{1} \\
k_{3} & k_{2} & k_{1}
\end{array}\right]_{\varepsilon}, \quad\left[\begin{array}{lll}
s_{3} & s_{2} & s_{1} \\
k_{3} & k_{2} & k_{1}
\end{array}\right]_{\varepsilon}=e^{-\varepsilon \sum_{i=1}^{3}\left|k_{i}\right|} \delta_{\varepsilon}\left(k_{3}-k_{2}-k_{1}\right) C_{s_{3}}\left[\begin{array}{ll}
s_{2} & s_{1} \\
k_{2} & k_{1}
\end{array}\right],
$$

where $\delta_{\varepsilon}(x)=\delta_{\varepsilon}(-x)$ is a symmetric regularization of the delta-distribution. Let us furthermore note that it suffices to check the invariance property on a dense subset $\mathscr{T}$ of the domain of $h_{1}$. Consider the matrix element

$$
\left\langle\left\langle\psi_{2}, k_{1}|\Delta(\mathrm{O})| \psi_{2}^{\prime}, k_{1}^{\prime}\right\rangle\right\rangle_{s_{2} s_{1}}:=\int_{\mathbb{R}} d k_{2} d k_{2}^{\prime} \psi\left(k_{2}\right) \psi^{\prime}\left(k_{2}^{\prime}\right)\left\langle\left\langle k_{2}, k_{1}|\Delta(\mathrm{O})| k_{2}^{\prime}, k_{1}^{\prime}\right\rangle\right\rangle_{s_{2} s_{1}},
$$

where $\psi_{2}, \psi_{2}^{\prime}$ are smooth functions with compact support. Assuming that $K_{\mathrm{O}}\left(k, k^{\prime} \mid s\right)$ has exponential decay w.r.t. $k$ and $k^{\prime}$ it is not difficult to show that the matrix element 
$\left\langle\left\langle\psi_{2}, k_{1}|\Delta(\mathrm{O})| \psi_{2}^{\prime}, k_{1}^{\prime}\right\rangle_{s_{2} s_{1}}\right.$ will also have exponential decay w.r.t. $k_{1}, k_{1}^{\prime}$ that can be made as large as one likes by choosing the subset $\mathscr{T} \subset \mathscr{A}_{h}$ appropriately.

Combining (4.2) and (4.4) leads to the following representation for the distributional matrix elements of $\left(\mathrm{id} \otimes \mathrm{h}_{1}\right) \Delta(\mathrm{O})$.

$$
\begin{aligned}
\left\langle\left\langle k_{2}\left|\left(\mathrm{id} \otimes \mathrm{h}_{1}\right) \Delta(\mathrm{O})\right| k_{2}^{\prime}\right\rangle\right\rangle_{s_{2}}= & \int_{\mathbb{R}^{+}} d \mathrm{~m}\left(\mathrm{~s}_{3}\right) \int_{\mathbb{R}} d k_{3} d k_{3}^{\prime} K_{\mathrm{O}}\left(k_{3}, k_{3}^{\prime} \mid s_{3}\right) \\
& \times \int_{\mathbb{R}^{+}} d \mathrm{~m}\left(\mathrm{~s}_{1}\right) \int_{\mathbb{R}} d k_{1} e^{-2 \pi Q k_{1}}\left(\left[\begin{array}{ccc}
s_{3} & s_{2} & s_{1} \\
k_{3} & k_{2} & k_{1}
\end{array}\right]\right)^{*}\left[\begin{array}{ccc}
s_{3} & s_{2} & s_{1} \\
k_{3}^{\prime} & k_{2}^{\prime} & k_{1}
\end{array}\right] .
\end{aligned}
$$

We are going to use the following result:

Proposition 1. - The following equation holds as an identity between tempered distributions:

$$
\begin{aligned}
\left.e^{2 \pi k_{3} Q} \int_{\mathbb{R}^{+}} d \mathrm{~m}\left(\mathrm{~s}_{1}\right) \int_{\mathbb{R}} d k_{1} e^{-2 \pi Q k_{1}}\left(\left[\begin{array}{lll}
s_{3} & s_{2} & s_{1} \\
k_{3} & k_{2} & k_{1}
\end{array}\right]\right)^{*} \begin{array}{lll}
s_{3} & s_{2} & s_{1} \\
k_{3}^{\prime} & k_{2}^{\prime} & k_{1}
\end{array}\right]= \\
\\
=\delta\left(k_{3}^{\prime}-k_{3}\right) \boldsymbol{\delta}\left(k_{2}^{\prime}-k_{2}\right) .
\end{aligned}
$$

Proof. - The proof of the proposition will be based on the following important symmetries of the Clebsch-Gordan kernel:

Lemma 8. - The Clebsch-Gordan kernel $\left[\begin{array}{lll}s_{3} & s_{2} & s_{1} \\ k_{3} & k_{2} & k_{1}\end{array}\right]_{\varepsilon}$ has the following symmetries.

$$
\begin{aligned}
& \left(\left[\begin{array}{lll}
s_{3} & s_{2} & s_{1} \\
k_{3} & k_{2} & k_{1}
\end{array}\right]_{\varepsilon}\right)^{*}=e^{+\pi Q\left(k_{1}-k_{3}\right)} e^{-\pi i h_{s_{2}}}\left[\begin{array}{ccc}
s_{1} & -s_{2} & s_{3} \\
-k_{1} & -k_{2} & -k_{3}
\end{array}\right]_{\varepsilon}, \\
& \left(\left[\begin{array}{lll}
s_{3} & s_{2} & s_{1} \\
k_{3} & k_{2} & k_{1}
\end{array}\right]_{\varepsilon}\right)^{*}=e^{-\pi Q\left(k_{2}-k_{3}\right)} e^{-\pi i h_{s_{1}}}\left[\begin{array}{ccc}
s_{2} & s_{3} & -s_{1} \\
-k_{2} & -k_{3} & -k_{1}
\end{array}\right]_{\varepsilon} .
\end{aligned}
$$

Proof. - One may verify directly that for $s_{i} \in \mathbb{R}, i=1,2,3$,

$$
\begin{aligned}
& \left(\left[\begin{array}{lll}
s_{3} & s_{2} & s_{1} \\
x_{3} & x_{2} & x_{1}
\end{array}\right]\right)^{*}=e^{-\pi i h_{s_{2}}}\left[\begin{array}{ccc}
s_{1} & -s_{2} & s_{3} \\
x_{1}^{*}-i \frac{Q}{2} & x_{2}^{*} & x_{3}^{*}-i \frac{Q}{2}
\end{array}\right], \\
& \left(\left[\begin{array}{lll}
s_{3} & s_{2} & s_{1} \\
x_{3} & x_{2} & x_{1}
\end{array}\right]\right)^{*}=e^{-\pi i h_{s_{1}}}\left[\begin{array}{ccc}
s_{2} & s_{3} & -s_{1} \\
x_{2}^{*}+i \frac{Q}{2} & x_{3}^{*}+i \frac{Q}{2} & x_{1}^{*}
\end{array}\right] .
\end{aligned}
$$

The Lemma follows by taking the Fourier-transformation of (4.8), taking into account that our regularization is compatible with the symmetry (4.7).

With the help of equation (4.7) we may rewrite the left hand side of 4.6) as follows:

$$
\lim _{\varepsilon \downarrow 0} e^{\pi\left(k_{3}-k_{3}^{\prime}\right) Q} \int_{\mathbb{R}^{+}} d \mathrm{~m}\left(\mathrm{~s}_{1}\right) \int_{\mathbb{R}} d k_{1}\left(\left[\begin{array}{ccc}
s_{1} & -s_{2} & s_{3} \\
-k_{1} & -k_{2} & -k_{3}
\end{array}\right]_{\varepsilon}\right)^{*}\left[\begin{array}{ccc}
s_{1} & -s_{2} & s_{3} \\
-k_{1} & -k_{2}^{\prime} & -k_{3}^{\prime}
\end{array}\right]_{\varepsilon}
$$

The proposition now follows by using the Fourier-transform of [PT2, Corollary 1].

Inserting (4.6) into (4.5) yields

$$
\left\langle\left\langle k_{2}\left|\left(\mathrm{id} \otimes \mathrm{h}_{1}\right) \Delta(\mathrm{O})\right| k_{2}^{\prime}\right\rangle\right\rangle_{s_{2}}=\delta\left(k_{2}-k_{2}^{\prime}\right) \int_{\mathbb{R}^{+}} d \mathrm{~m}\left(s_{3}\right) \int_{\mathbb{R}} d k_{3} e^{-2 \pi Q k_{3}} K_{\mathrm{O}}\left(k_{3}, k_{3} \mid s_{3}\right) .
$$

Recognizing the definition of the Haar-measure on the right hand side completes the proof of the left invariance property of $h_{1}$. 
To prove the property (ii) in Theorem 2 we observe that definition (2.18) can be rewritten as

$$
\mathrm{h}_{1}(\mathrm{O})=\int_{0}^{\infty} d \mathrm{~m}(s) \operatorname{Tr}\left(\mathrm{K}^{2} \tilde{\mathrm{K}}^{2} \mathrm{O}_{s}\right) .
$$

Now it is straightforward to verify the first formula in (2.21) for $X=E, F, K$ using the definition of the adjoint action, the relations (2.1), and the cyclicity of trace. For instance, $\operatorname{Tr}\left(\mathrm{K}^{2} \tilde{\mathrm{K}}^{2} \mathrm{ad}_{\mathrm{K}}^{1}\left(\mathrm{O}_{s}\right)\right)=\operatorname{Tr}\left(\mathrm{K}^{2} \tilde{\mathrm{K}}^{2} \mathrm{KO}_{s} \mathrm{~K}^{-1}\right)=\operatorname{Tr}\left(\mathrm{K}^{2} \tilde{\mathrm{K}}^{2} \mathrm{O}_{s}\right), \operatorname{Tr}\left(\mathrm{K}^{2} \tilde{\mathrm{K}}^{2} \operatorname{ad}_{\mathrm{E}}^{1}\left(\mathrm{O}_{s}\right)\right)=$ $\operatorname{Tr}\left(\mathrm{K}^{2} \tilde{\mathrm{K}}^{2}\left(\mathrm{EO}_{s} \mathrm{~K}^{-1}-q \mathrm{~K}^{-1} \mathrm{O}_{s} \mathrm{E}\right)\right)=\operatorname{Tr}\left((\mathrm{KE}-q \mathrm{EK}) \tilde{\mathrm{K}}^{2} \mathrm{O}_{s}\right)=0$. Further, we notice that $\mathrm{h}_{1}\left(\operatorname{ad}_{X Y}^{1}(\mathrm{O})\right)=\mathrm{h}_{1}\left(\operatorname{ad}_{X}^{1}\left(\operatorname{ad}_{Y}^{1}(\mathrm{O})\right)\right)=\mathrm{h}_{1}(\mathrm{O}) \varepsilon(\mathrm{X}) \varepsilon(\mathrm{Y})=\mathrm{h}_{1}(\mathrm{O}) \varepsilon(\mathrm{XY})$. Together with the linearity of trace this implies that (2.21) extends to any element of $U_{q}(\mathfrak{s l}(2, \mathbb{R}))$.

4.3. Proof of Theorem 3. - Let us adopt the following notations: $X_{1} \equiv X \otimes 1 \otimes 1$, $\mathrm{X}_{2} \equiv 1 \otimes \mathrm{X} \otimes 1$, and $\mathrm{X}_{3} \equiv 1 \otimes 1 \otimes \mathrm{X}$.

Property (i) First, we compute with the help of Lemma 5:

$$
\begin{aligned}
& q^{\mathrm{H} \otimes \mathrm{H}}\left(\mathrm{E}_{1} \mathrm{~F}_{2}\right)^{i t} q^{\mathrm{H} \otimes \mathrm{H}}\left(\mathrm{K}_{1}^{-1} \mathrm{E}_{2}\right)-\left(\mathrm{K}_{1} \mathrm{E}_{2}\right) q^{\mathrm{H} \otimes \mathrm{H}}\left(\mathrm{E}_{1} \mathrm{~F}_{2}\right)^{i t} q^{\mathrm{H} \otimes \mathrm{H}} \\
& =q^{\mathrm{H} \otimes \mathrm{H}}\left[\left(\mathrm{E}_{1} \mathrm{~F}_{2}\right)^{i t}, \mathrm{E}_{2}\right] q^{\mathrm{H} \otimes \mathrm{H}} \stackrel{(3.25)}{=}-q^{\mathrm{H} \otimes \mathrm{H}}\left([i t]_{q}\left[2 \mathrm{H}_{2}+i t-1\right]_{q} \mathrm{E}_{1}^{i t} \mathrm{~F}_{2}^{i t-1}\right) q^{\mathrm{H} \otimes \mathrm{H}} .
\end{aligned}
$$

Next, we find

$$
\begin{aligned}
& q^{\mathrm{H} \otimes \mathrm{H}}\left(\mathrm{E}_{1} \mathrm{~F}_{2}\right)^{i t} q^{\mathrm{H} \otimes \mathrm{H}}\left(\mathrm{E}_{1} \mathrm{~K}_{2}\right)-\left(\mathrm{E}_{1} \mathrm{~K}_{2}^{-1}\right) q^{\mathrm{H} \otimes \mathrm{H}}\left(\mathrm{E}_{1} \mathrm{~F}_{2}\right)^{i t} q^{\mathrm{H} \otimes \mathrm{H}} \\
& =q^{\mathrm{H} \otimes \mathrm{H}}\left(\left(\mathrm{E}_{1} \mathrm{~F}_{2}\right)^{i t} \mathrm{E}_{1} \mathrm{~K}_{2}^{2}-\mathrm{E}_{1} \mathrm{~K}_{2}^{-2}\left(\mathrm{E}_{1} \mathrm{~F}_{2}\right)^{i t}\right) q^{\mathrm{H} \otimes \mathrm{H}} \\
& =q^{\mathrm{H} \otimes \mathrm{H}}\left(\left(2 i q^{i t} \sin \pi b^{2}\right)\left[2 \mathrm{H}_{2}+i t\right]_{q} \mathrm{E}_{1}^{i t+1} \mathrm{~F}_{2}^{i t}\right) q^{\mathrm{H} \otimes \mathrm{H}} .
\end{aligned}
$$

Let us write down the integral representation (3.28) of $\mathrm{R}$ in the following form

$$
\mathrm{R}=\int_{\mathbb{R}} d t \rho(t) q^{\mathrm{H} \otimes \mathrm{H}}(\mathrm{E} \otimes \mathrm{F})^{i t} q^{\mathrm{H} \otimes \mathrm{H}},
$$

where $\rho(t) \equiv b \frac{e^{-\pi i b^{2} t^{2}}}{G_{b}(Q+i b t)}\left(2 \sin \pi b^{2}\right)^{2 i t}$. Observe that (3.3) implies that $\rho(t)$ satisfies the following functional equation:

$$
[i t+1]_{q} \rho(t-i)=\left(2 i q^{i t} \sin \pi b^{2}\right) \rho(t) .
$$

Adding (4.10) with (4.11), we derive

$$
\begin{aligned}
& \mathrm{R} \Delta(\mathrm{E})-\Delta^{\prime}(\mathrm{E}) \mathrm{R}=\mathrm{R}\left(\mathrm{E}_{1} \mathrm{~K}_{2}+\mathrm{K}_{1}^{-1} \mathrm{E}_{2}\right)-\left(\mathrm{E}_{1} \mathrm{~K}_{2}^{-1}+\mathrm{K}_{1} \mathrm{E}_{2}\right) \mathrm{R} \\
& \stackrel{4.12)}{=} \int_{\mathbb{R}} d t q^{\mathrm{H} \otimes \mathrm{H}} \rho(t)\left(\left(2 i q^{i t} \sin \pi b^{2}\right)\left[2 \mathrm{H}_{2}+i t\right]_{q} \mathrm{E}_{1}^{i t+1} \mathrm{~F}_{2}^{i t}\right. \\
& \left.-[i t]_{q}\left[2 \mathrm{H}_{2}+i t-1\right]_{q} \mathrm{E}_{1}^{i t} \mathrm{~F}_{2}^{i t-1}\right) q^{\mathrm{H} \otimes \mathrm{H}} \\
& =\int_{\mathbb{R}} d t q^{\mathrm{H} \otimes \mathrm{H}}\left(\left(2 i q^{i t} \sin \pi b^{2}\right) \rho(t)\right. \\
& \left.-[i t+1]_{q} \rho(t-i)\right)\left[2 \mathrm{H}_{2}+i t\right]_{q} \mathrm{E}_{1}^{i t+1} \mathrm{~F}_{2}^{i t} q^{\mathrm{H} \otimes \mathrm{H} \stackrel{4.13}{=} 0 .}
\end{aligned}
$$


Thus, we have proven (2.24) for $\mathrm{X}=\mathrm{E}$. The proof for $\mathrm{F}$ goes along the same lines with the help of the second formula in (3.25). And for $\mathrm{K}$ the proof is trivial because $\Delta(\mathrm{K})$ commutes with $(\mathrm{E} \otimes \mathrm{F})^{i t}$.

Property (ii) Recall that the rescaled generators $\mathrm{e}_{b}$ and $\mathrm{f}_{b}$ were introduced before Lemma 4. To prove the first formula in (2.25), we use the quantum exponential relation (3.26) from Lemma 6 with identification $u=\mathrm{e}_{1} \mathrm{~K}_{2}^{-1} \mathrm{f}_{3}$ and $v=\mathrm{e}_{1} \mathrm{f}_{2} \mathrm{~K}_{3}$,

$$
\begin{array}{rll}
(\mathrm{id} \otimes \Delta) \mathrm{R} & \stackrel{2.23}{=}(\mathrm{id} \otimes \Delta)\left(q^{\mathrm{H}_{1} \mathrm{H}_{2}} g_{b}\left(\mathrm{e}_{1} \mathrm{f}_{2}\right) q^{\mathrm{H}_{1} \mathrm{H}_{2}}\right) \\
& \stackrel{2.11}{=} & q^{\mathrm{H}_{1} \mathrm{H}_{2}+\mathrm{H}_{1} \mathrm{H}_{3}} g_{b}\left(\mathrm{e}_{1} \mathrm{f}_{2} \mathrm{~K}_{3}+\mathrm{e}_{1} \mathrm{~K}_{2}^{-1} \mathrm{f}_{3}\right) q^{\mathrm{H}_{1} \mathrm{H}_{2}+\mathrm{H}_{1} \mathrm{H}_{3}} \\
& q^{\mathrm{H}_{1} \mathrm{H}_{2}+\mathrm{H}_{1} \mathrm{H}_{3}} g_{b}\left(\mathrm{e}_{1} \mathrm{~K}_{2}^{-1} \mathrm{f}_{3}\right) g_{b}\left(\mathrm{e}_{1} \mathrm{f}_{2} \mathrm{~K}_{3}\right) q^{\mathrm{H}_{1} \mathrm{H}_{2}+\mathrm{H}_{1} \mathrm{H}_{3}} \\
& q^{\mathrm{H}_{1} \mathrm{H}_{3}} g_{b}\left(\mathrm{e}_{1} \mathrm{f}_{3}\right) q^{\mathrm{H}_{1} \mathrm{H}_{3}} q^{\mathrm{H}_{1} \mathrm{H}_{2}} g_{b}\left(\mathrm{e}_{1} \mathrm{f}_{2}\right) q^{\mathrm{H}_{1} \mathrm{H}_{2}}=\mathrm{R}_{13} \mathrm{R}_{12} .
\end{array}
$$

The second formula in 2.25 ) is proved in the same way.

Property (iii) First, we derive

$$
\begin{aligned}
& (\sigma \otimes \mathrm{id})\left(q^{\mathrm{H}_{1} \mathrm{H}_{2}}\left(\mathrm{E}_{1} \mathrm{~F}_{2}\right)^{i t} q^{\mathrm{H}_{1} \mathrm{H}_{2}}\right)=(\sigma \otimes \mathrm{id})\left(q^{\mathrm{H}_{1} \mathrm{H}_{2}}\left(\mathrm{E}_{1}\right)^{i t} q^{\mathrm{H}_{1}\left(\mathrm{H}_{2}+i t\right)}\right)\left(\mathrm{F}_{2}\right)^{i t} \\
& =q^{-\mathrm{H}_{1}\left(\mathrm{H}_{2}+i t\right)}\left(-q \mathrm{E}_{1}\right)^{i t} q^{-\mathrm{H}_{1} \mathrm{H}_{2}}\left(\mathrm{~F}_{2}\right)^{i t}=q^{-\mathrm{H}_{1} \mathrm{H}_{2}} q^{-i t \mathrm{H}_{1}}\left(-q \mathrm{E}_{1}\right)^{i t} q^{i t \mathrm{H}_{1}}\left(\mathrm{~F}_{2}\right)^{i t} q^{-\mathrm{H}_{1} \mathrm{H}_{2}} \\
& =e^{\pi b\left(i b t^{2}-Q t\right)} q^{-\mathrm{H}_{1} \mathrm{H}_{2}}\left(\mathrm{E}_{1} \mathrm{~F}_{2}\right)^{i t} q^{-\mathrm{H}_{1} \mathrm{H}_{2}}
\end{aligned}
$$

This means that, acting with $(\sigma \otimes$ id $)$ on the r.h.s. of (3.28), we obtain the r.h.s. of (3.29). Thus, we have proven the first formula in (2.25). The second formula is verified analogously. Finally, acting with $(\mathrm{id} \otimes \sigma)$ on the last line in (4.14) and performing similar manipulations, we find that

$$
(\mathrm{id} \otimes \sigma)\left(e^{\pi b\left(i b t^{2}-Q t\right)} q^{-\mathrm{H}_{1} \mathrm{H}_{2}}\left(\mathrm{E}_{1} \mathrm{~F}_{2}\right)^{i t} q^{-\mathrm{H}_{1} \mathrm{H}_{2}}\right)=q^{\mathrm{H}_{1} \mathrm{H}_{2}}\left(\mathrm{E}_{1} \mathrm{~F}_{2}\right)^{i t} q^{\mathrm{H}_{1} \mathrm{H}_{2}}
$$

which together with (4.14) implies the last formula in (2.26).

4.4. Proof of Theorem 5: R-operator the highest weight representations. - We first need to discuss the analytic continuation of

$$
\left\langle\delta_{k_{2}} \otimes \delta_{k_{1}}, \mathrm{R}_{s_{2} s_{1}} f\right\rangle=\int_{\mathbb{R}} d k_{2}^{\prime} d k_{1}^{\prime} \tilde{\mathrm{R}}\left(k_{2}, k_{1} \mid k_{2}^{\prime}, k_{2}^{\prime}\right) f\left(k_{2}^{\prime}, k_{2}^{\prime}\right)
$$

to the values $k_{1}=-s_{1}+i\left(\frac{Q}{2}+n b\right), n \in \mathbb{Z}^{\geq 0}$. To begin with, one may trivially perform e.g. the integral over $k_{2}^{\prime}$ to get an expression of the form

$$
\left\langle\delta_{k_{2}} \otimes \delta_{k_{1}}, \mathrm{R}_{s_{2} s_{1}} f\right\rangle=\int_{\mathbb{R}} d k_{1}^{\prime} \tilde{\mathrm{R}}_{k}\left(k_{1} \mid k_{1}^{\prime}\right) f\left(k-k_{1}^{\prime}, k_{1}^{\prime}\right),
$$

where $k=k_{2}+k_{1}$. The analytic continuation of (4.16) to $k_{1}=-s_{1}+i\left(\frac{Q}{2}+n b\right)$ can be defined by deforming the contour of integration over $k_{1}^{\prime}, \mathbb{R}$, in (4.16) into $\mathbb{R}+i\left(\frac{Q}{2}+\right.$ $n b)+i 0$ plus a sum of small circles around the poles from the factor $w_{b}^{-1}\left(s_{1}+k_{1}^{\prime}\right)$ in $\tilde{\mathrm{R}}_{k}\left(k_{1} \mid k_{1}^{\prime}\right)$ that lie between $\mathbb{R}$ and $\mathbb{R}+i\left(\frac{Q}{2}+n b\right)+i 0$.

We are now in the position to take the limit $k_{1} \rightarrow-s_{1}+i\left(\frac{Q}{2}+n b\right)$. The factor $w_{b}\left(s_{1}+k_{1}\right)$ that appears in $\tilde{\mathrm{R}}_{k}\left(k_{1} \mid k_{1}^{\prime}\right)$, cf. (2.30), makes most of the terms vanish 
except for the terms from the poles at $k_{1}^{\prime}=-s_{1}+i\left(\frac{Q}{2}+b n^{\prime}\right), 0 \leq n^{\prime} \leq n$. The resulting expression is of the following form.

$$
\mathrm{R}_{s_{2} s_{1}}^{t} \delta_{k_{2}} \otimes \delta_{k_{1}}=\left.\sum_{l=0}^{n} G_{l} e^{-\pi i\left(\left(k_{1}-i b l\right) k_{2}+k_{1} k_{2}^{\prime}\right)} \frac{w_{n-l}}{w_{n}} \frac{w_{b}\left(s_{2}-k_{2}\right)}{w_{b}\left(s_{2}-k_{2}^{\prime}\right)}\right|_{k_{2}^{\prime}=k_{2}+i b l}
$$

where $G_{n}:=\operatorname{Res}_{x=n b} G_{b}^{-1}(Q+x)$ and $w_{n}=\operatorname{Res}_{x=i \frac{Q}{2}+i n b} w_{b}^{-1}(x)$.

It remains to calculate the relevant residues. It is easy to derive from (3.2)-(3.4) that

$$
G_{b}(x) G_{b}(-x)=-\frac{e^{\pi i x^{2}}}{4 \sin \pi b x \sin \pi b^{-1} x} .
$$

Hence $\lim _{x \rightarrow 0}\left(x G_{b}(x)\right)^{2}=(2 \pi)^{-2}$. In fact, using the modular property of the Dedekind $\eta$-function, it is straightforward to compute the limit directly for the product representation (3.8) (as was done in [Sh] $)$; which yields

$$
\lim _{x \rightarrow 0} x G_{b}(x)=\frac{1}{2 \pi} \text {. }
$$

Hence, taking into account the properties (3.2)-(3.4), we find that

$$
\operatorname{Res} \frac{1}{G_{b}(Q+z)}=-\frac{1}{2 \pi} \prod_{k=1}^{n}\left(1-q^{2 k}\right)^{-1} \prod_{l=1}^{m}\left(1-\tilde{q}^{-2 l}\right)^{-1} \quad \text { at } z=n b+m b^{-1},
$$

where $n, m \in \mathbb{Z}^{\geq 0}$ and $\tilde{q}=e^{-i \pi b^{-2}}$.

To complete the proof of Theorem 5 is now the matter of a straightforward calculation using the functional relation (3.11), formula (4.20), as well as (2.31).

4.5. Proof of Theorem 6. - Let us first note that the left hand side of (2.33) satisfies the intertwining property

$$
\mathrm{C}\left[s_{3} \mid s_{1}, s_{2}\right] \mathrm{B}_{s_{2} s_{1}} \circ\left(\pi_{s_{2}} \otimes \pi_{s_{1}}\right) \circ \Delta(X)=\pi_{s_{3}}(X) \circ \mathrm{C}\left[s_{3} \mid s_{1}, s_{2}\right] \mathrm{B}_{s_{2} s_{1}} .
$$

A unitary operator that maps $\mathscr{P}_{s_{2}} \otimes \mathscr{P}_{s_{1}} \rightarrow \mathscr{P}_{s_{3}}$ and satisfies (2.14) must be proportional to $\mathrm{C}\left[s_{3} \mid s_{2}, s_{1}\right]$. This is a consequence of the analysis used to prove Theorem 2 in [PT2]. It follows that there exists a function $\Omega\left(s_{3} \mid s_{2}, s_{1}\right)$ such that the statement of Theorem 6 holds. We are left with the task to calculate $\Omega\left(s_{3} \mid s_{2}, s_{1}\right)$ explicitly.

To this aim let us note that Theorem 6 is equivalent to an identity between meromorphic functions. To write this identity down, let us assume that $\mathscr{P}_{s_{2}} \otimes \mathscr{P}_{s_{1}}$ is realized by functions $\tilde{\psi}\left(k_{2}, k_{1}\right)$. C $\left[s_{3} \mid s_{1}, s_{2}\right]$ is then realized as an integral operator:

$$
\left(\mathrm{C}\left[s_{3} \mid s_{1}, s_{2}\right] \tilde{\psi}\right)\left(k_{3}\right)=\int_{\mathbb{R}} d k_{2} d k_{1}\left[\begin{array}{ccc}
s_{3} & s_{2} & s_{1} \\
k_{3} & k_{2} & k_{1}
\end{array}\right] \tilde{\psi}\left(k_{2}, k_{1}\right) .
$$

The explicit expression for the distributional kernel $\left[\begin{array}{ccc}s_{3} & s_{2} & s_{1} \\ k_{3} & k_{2} & k_{1}\end{array}\right]$ can be found in Appendix A. For the moment it will be enough to note that it can be factorized as

$$
\left[\begin{array}{lll}
s_{3} & s_{2} & s_{1} \\
k_{3} & k_{2} & k_{1}
\end{array}\right]=\delta\left(k_{1}+k_{2}-k_{3}\right) C_{s_{3}}\left[\begin{array}{ll}
s_{2} & s_{1} \\
k_{2} & k_{1}
\end{array}\right]
$$


where $C_{S_{3}}\left[\begin{array}{ll}S_{2} & s_{1} \\ k_{2} & k_{1}\end{array}\right]$ is a meromorphic function by Lemma 20 of [PT2]. It is then easy to see that Theorem 6 is equivalent to the identity

$$
\int_{\mathbb{R}} d k_{2} d k_{1}\left[\begin{array}{lll}
s_{3} & s_{1} & s_{2} \\
k_{3} & k_{1} & k_{2}
\end{array}\right] \tilde{R}_{s_{2} s_{1}}\left(k_{2}, k_{1} \mid k_{2}^{\prime}, k_{1}^{\prime}\right)=\Omega\left(s_{3} \mid s_{2}, s_{1}\right)\left[\begin{array}{lll}
s_{3} & s_{2} & s_{1} \\
k_{3} & k_{2}^{\prime} & k_{1}^{\prime}
\end{array}\right] .
$$

In order to see that equation (4.23) is indeed equivalent to an identity between meromorphic functions let us note that (4.22) and (2.30) allow one to split off the distributional factors. What remains on the left hand side is a convolution of two meromorphic functions, so is itself meromorphic (cf. Lemma 3 in [एPT2]).

Let us note that both sides of (4.23) have a pole at $k_{2}^{\prime}=-s_{2}+i \frac{Q}{2}$. In the case of the right hand side this is a consequence of Lemma 20 of [PT2]. Concerning the left hand side of (4.23) one may as in the proof of Lemma 3 of [PT2] identify the above-mentioned pole as the consequence of the pinching of the contour of integration by a collision of two poles of the integrand. First we have the pole of $\left[\begin{array}{lll}s_{3} & s_{1} & s_{2} \\ k_{3} & k_{1} & k_{2}\end{array}\right]$ at $k_{2}=-s_{2}+i \frac{Q}{2}$. Second let us note that the factor $G_{b}^{-1}\left(Q+i\left(k_{1}^{\prime}-k_{1}\right)\right)$ appearing in $\tilde{\mathrm{R}}_{s_{2} s_{1}}\left(k_{2}, k_{1} \mid k_{2}^{\prime}, k_{1}^{\prime}\right)$ produces a pole at $k_{2}=k_{2}^{\prime}$ if one takes into account that $\tilde{\mathrm{R}}_{s_{2} s_{1}}\left(k_{2}, k_{1} \mid k_{2}^{\prime}, k_{1}^{\prime}\right)$ has support only for $k_{1}^{\prime}-k_{1}=k_{2}-k_{2}^{\prime}$. The residue of the resulting pole on the left hand side of (4.23) is simply given by the product of the relevant residues of $\left[\begin{array}{lll}s_{3} & s_{1} & s_{2} \\ k_{3} & k_{1} & k_{2}\end{array}\right]$ and $\tilde{\mathrm{R}}_{s_{2} s_{1}}\left(k_{2}, k_{1} \mid k_{2}^{\prime}, k_{1}^{\prime}\right)$ respectively.

The equality of the residues of the two sides of equation (4.23) implies the following identity:

$$
\operatorname{Res}_{k_{2}=-s_{2}+i \frac{Q}{2}} C_{s_{3}}\left[\begin{array}{ll}
s_{1} & s_{2} \\
k_{1} & k_{2}
\end{array}\right] e^{\pi Q k_{1}} e^{2 \pi i s_{2} k_{1}}=\Omega\left(s_{3} \mid s_{2}, s_{1}\right) \underset{k_{2}=-s_{2}+i \frac{Q}{2}}{\operatorname{Res}} C_{s_{3}}\left[\begin{array}{ll}
s_{2} & s_{1} \\
k_{2} & k_{1}
\end{array}\right]
$$

By evaluating the relevant residues we may therefore calculate $\Omega\left(s_{3} \mid s_{2}, s_{1}\right)$.

Lemma 9. -

$$
\begin{aligned}
& 2 \pi i \operatorname{Res} C_{s_{3}}\left[\begin{array}{ll}
s_{1} & s_{2} \\
k_{1} & k_{2}
\end{array}\right]=e^{-\frac{\pi i}{2}\left(h_{s_{3}}-h_{s_{2}}-h_{s_{1}}\right)} e^{-\frac{\pi}{2} Q k_{1}} e^{-\pi i s_{2} k_{1}} \frac{w_{b}\left(k_{1}-s_{1}\right) w_{b}\left(s_{3}+s_{1}-s_{2}\right)}{w_{b}\left(k_{1}+s_{3}-s_{2}+i \frac{Q}{2}\right)}, \\
& 2 \pi i \operatorname{Res} C_{s_{3}=-s_{2}+i \frac{2}{2}}\left[\begin{array}{ll}
s_{2} & s_{1} \\
k_{2} & k_{1}
\end{array}\right]=e^{+\frac{\pi i}{2}\left(h_{s_{3}}-h_{s_{2}}-h_{s_{1}}\right)} e^{+\frac{\pi}{2} Q k_{1}} e^{+\pi i s_{2} k_{1}} \frac{w_{b}\left(k_{1}-s_{1}\right) w_{b}\left(s_{3}+s_{1}-s_{2}\right)}{w_{b}\left(k_{1}+s_{3}-s_{2}+i \frac{Q}{2}\right)} .
\end{aligned}
$$

Proof. - In order to exhibit the singular behavior of $C_{s_{3}}\left[\begin{array}{ll}s_{1} & s_{2} \\ k_{1} & k_{2}\end{array}\right]$ near $k_{2}=-s_{2}+i \frac{Q}{2}$ one may deform the contour of integration in (A.6) into the union of a small circle around the pole of the integrand at $s=0$ and a contour that separates the pole at $s=0$ from all the other poles in the upper half-plane, approaching asymptotically $\pm i \infty$. The contribution from the residue of the pole at $s=0$ exhibits the pole at $k_{2}=-s_{2}+i \frac{Q}{2}$ explicitly, whereas the rest is nonsingular.

Similarly, to analyze the singular behavior of $C_{s_{3}}\left[\begin{array}{ll}s_{2} & s_{1} \\ k_{2} & k_{1}\end{array}\right]$ near $k_{2}=-s_{2}+i \frac{Q}{2}$ one needs to deform the contour in A.6 into a small circle around the pole at $s=-R_{3}$ together with a contour separating that pole from all the other poles in the lower half plane.

It is then straightforward to calculate the values of the corresponding residues from (A.6). 


\section{A}

\section{The Clebsch-Gordan coefficients for the modular double}

Definition 2. - Define a distributional kernel $\left[\begin{array}{ccc}s_{3} & s_{2} & s_{1} \\ x_{3} & x_{2} & x_{1}\end{array}\right]$ (the "Clebsch-Gordan coefficients") by an expression of the form

$$
\left[\begin{array}{lll}
s_{3} & s_{2} & s_{1} \\
x_{3} & x_{2} & x_{1}
\end{array}\right] \equiv \lim _{\varepsilon \downarrow 0}\left[\begin{array}{lll}
s_{3} & s_{2} & s_{1} \\
x_{3} & x_{2} & x_{1}
\end{array}\right]_{\varepsilon}
$$

where the meromorphic function $\left[\begin{array}{lll}s_{3} & s_{2} & s_{1} \\ x_{3} & x_{2} & x_{1}\end{array}\right]_{\varepsilon}$ is defined as

$$
\begin{aligned}
{\left[\begin{array}{ccc}
-s_{3} & s_{2} & s_{1} \\
x_{3} & x_{2} & x_{1}
\end{array}\right]_{\varepsilon}=e^{-\frac{\pi i}{2}\left(h_{s_{3}}-h_{s_{2}}-h_{s_{1}}\right)} } & \\
& \times D_{b}\left(\sigma_{32} ; y_{32}+i \varepsilon\right) D_{b}\left(\sigma_{31} ; y_{31}+i \varepsilon\right) D_{b}\left(\sigma_{21} ; y_{21}+i \varepsilon\right),
\end{aligned}
$$

$h_{s}=s^{2}+\frac{1}{4} Q^{2}$, the distribution $D_{b}(\sigma ; y)$ is defined in terms of the function $w_{b}(y)$ as

$$
D_{b}(\sigma ; y)=\frac{w_{b}\left(y-\frac{i}{2} Q\right)}{w_{b}(y+s)}
$$

and the coefficients $y_{j i}, \beta_{j i}, j>i \in\{1,2,3\}$ are given by

$$
\begin{array}{ll}
y_{32}=x_{2}-x_{3}+\frac{1}{2}\left(s_{3}+s_{2}\right) & \sigma_{32}=s_{1}-s_{2}-s_{3} \\
y_{31}=x_{3}-x_{1}+\frac{1}{2}\left(s_{3}+s_{1}\right) & \sigma_{31}=s_{2}-s_{3}-s_{1} \\
y_{21}=x_{2}-x_{1}+\frac{1}{2}\left(s_{2}+s_{1}-2 s_{3}\right) & \sigma_{21}=s_{3}-s_{2}-s_{1} .
\end{array}
$$

It is often useful to consider the Fourier-transform of the b-Clebsch-Gordan symbols defined by

$$
\left[\begin{array}{ccc}
s_{3} & s_{2} & s_{1} \\
-k_{3} & k_{2} & k_{1}
\end{array}\right]=\int_{\mathbb{R}} d x_{3} d x_{2} d x_{1} e^{2 \pi i \sum_{l=1}^{3} k_{l} x_{l}}\left[\begin{array}{ccc}
s_{3} & s_{2} & s_{1} \\
x_{3} & x_{2} & x_{1}
\end{array}\right]
$$

The distribution $\left[\begin{array}{lll}s_{3} & s_{2} & s_{1} \\ k_{3} & k_{2} & k_{1}\end{array}\right]$ can be factorized as

$$
\left[\begin{array}{lll}
s_{3} & s_{2} & s_{1} \\
k_{3} & k_{2} & k_{1}
\end{array}\right]=\delta\left(k_{1}+k_{2}-k_{3}\right) C_{s_{3}}\left[\begin{array}{ll}
s_{2} & s_{1} \\
k_{2} & k_{1}
\end{array}\right]
$$

where $C_{s_{3}}\left[\begin{array}{ll}s_{2} & s_{1} \\ k_{2} & k_{1}\end{array}\right]$ is a meromorphic function. A straightforward calculation using [PT2, Lemma 15] yields the following expression:

$$
C_{-s_{3}}\left[\begin{array}{ll}
s_{2} & s_{1} \\
k_{2} & k_{1}
\end{array}\right]=\frac{e^{-\frac{\pi i}{2} \beta_{21} \beta} e^{\frac{Q}{2} \pi\left(k_{1}-k_{2}\right)} e^{\pi i\left(k_{1} s_{2}-k_{2} s_{1}\right)}}{w_{b}\left(\sigma_{32}\right) w_{b}\left(\sigma_{31}\right) w_{b}\left(\sigma_{21}\right)} \int_{\mathbb{R}+i 0} d s e^{-\pi s \beta} \prod_{l=1}^{3} \frac{w_{b}\left(s+R_{l}\right)}{w_{b}\left(s+S_{l}\right)}
$$

where we used the abbreviations $\beta=\frac{Q}{2}+i\left(s_{1}+s_{2}+s_{3}\right), \beta_{21}=\frac{Q}{2}+i\left(s_{1}+s_{2}-s_{3}\right)$ and

$$
\begin{array}{ll}
R_{1}=-s_{2}+k_{2}, & S_{1}=i \frac{Q}{2}+R_{1}-\sigma_{32}, \\
R_{2}=-s_{1}-k_{1}, & S_{2}=i \frac{Q}{2}+R_{2}-\sigma_{31}, \\
R_{3}=s_{3}-s_{2}-s_{1}, & S_{3}=i \frac{Q}{2},
\end{array}
$$

The analytic properties of $\left[\begin{array}{lll}s_{3} & s_{2} & s_{1} \\ k_{3} & k_{2} & k_{1}\end{array}\right]$ can be summarized as follows: 
Lemma 10. - (Lemma 20 in [PT2]) $\left[\begin{array}{lll}s_{3} & s_{2} & s_{1} \\ k_{3} & k_{2} & k_{1}\end{array}\right]$ depends meromorphically on all of its arguments, with poles at $\pm i k_{i}=\frac{Q}{2}+i s_{i}+n b+m b^{-1}, n, m \in \mathbb{Z}^{\geq 0}, i=1,2,3$ only.

\section{B}

\section{Quantum exponential function and $b$-binomial coefficient}

The definition (2.22) and the property (3.3) imply that the function $g_{b}(x)$ obeys the following functional equation

$$
g_{b}(q x)=(1+x)^{-1} g_{b}\left(q^{-1} x\right) .
$$

For a pair of Weyl-type variables, $u v=q^{2} v u$, a consequence of (B.1) is

$$
u+v=g_{b}\left(q u^{-1} v\right) u\left(g_{b}\left(q u^{-1} v\right)\right)^{-1}=\left(g_{b}\left(q u v^{-1}\right)\right)^{-1} v g_{b}\left(q u v^{-1}\right) .
$$

It is now obvious that

$$
g_{b}(u+v)=g_{b}\left(q u^{-1} v\right) g_{b}(u)\left(g_{b}\left(q u^{-1} v\right)\right)^{-1}=\left(g_{b}\left(q u v^{-1}\right)\right)^{-1} g_{b}(v) g_{b}\left(q u v^{-1}\right) .
$$

These relations allow us to prove the equivalence of 3.26 ) and (3.27) stated in Lemma 6. For instance, let us show that (3.27) together with the first relation in $(\mathrm{B.3})$ implies (3.26). Introduce $V \equiv q u^{-1} v$. Notice that $V=e^{b \tilde{B}}$ where $\tilde{B}=B-A$ so that $[A, \tilde{B}]=2 \pi i$. Then we have

$$
g_{b}(u+v) \stackrel{\sqrt[B .3]{-}}{=} g_{b}(V) g_{b}(u)\left(g_{b}(V)\right)^{-1 \stackrel{(3.27)}{=}} g_{b}(u) g_{b}\left(q^{-1} u V\right)=g_{b}(u) g_{b}(v) \text {. }
$$

The inverse implication, $(3.27) \Rightarrow(3.26)$, is proven similarly.

Now we want to prove $(3.26)$. First, we represent $(u+v)^{i t}$ in an integral form:

$$
\begin{aligned}
& (u+v) \stackrel{\text { B.2. }}{=} g_{b}\left(q u^{-1} v\right) u^{i t}\left(g_{b}\left(q u^{-1} v\right)\right)^{-1} \\
& \quad b_{\mathbb{R}} d \tau_{1} d \tau_{2} e^{\pi b Q\left(\tau_{1}-\tau_{2}\right)} \frac{G_{b}\left(-i b \tau_{1}\right)}{G_{b}\left(Q+i b \tau_{2}\right)}\left(q u^{-1} v\right)^{i \tau_{1}} u^{i t}\left(q u^{-1} v\right)^{i \tau_{2}} \\
& \quad=b^{2} \int_{\mathbb{R}} d \tau_{1} d \tau_{2} e^{\pi b Q\left(\tau_{1}-\tau_{2}\right)-i \pi b^{2}\left(\tau_{1}+\tau_{2}\right)^{2}+2 i \pi b^{2} t \tau_{1}} \frac{G_{b}\left(-i b \tau_{1}\right)}{G_{b}\left(Q+i b \tau_{2}\right)} u^{i\left(t-\tau_{1}-\tau_{2}\right)} v^{i\left(\tau_{1}+\tau_{2}\right)} \\
& \quad=b^{2} \int_{\mathbb{R}} d \tau d \tau_{2} e^{\pi b \tau(Q+2 i b t)-i \pi b^{2} \tau^{2}-2 \pi b \tau_{2}(Q+i b t)} \frac{G_{b}\left(i b \tau_{2}-i b \tau\right)}{G_{b}\left(Q+i b \tau_{2}\right)} u^{i(t-\tau)} v^{i \tau}
\end{aligned}
$$

where we introduced $\tau \equiv \tau_{1}+\tau_{2}$. Computing the integral over $\tau_{2}$ with the help of (3.30), we derive an analogue of the binomial formula:

$$
(u+v)^{i t}=b \int_{\mathbb{R}+i 0} d \tau\left(\begin{array}{l}
t \\
\tau
\end{array}\right)_{b} u^{i(t-\tau)} v^{i \tau}
$$

where the $b$-binomial coefficient is given by

$$
\left(\begin{array}{l}
t \\
\tau
\end{array}\right)_{b}=\frac{e^{2 \pi i b^{2} \tau(t-\tau)} G_{b}(Q+i b t)}{G_{b}(Q+i b t-i b \tau) G_{b}(Q+i b \tau)} .
$$


We see that the function $G_{b}$ is a $b$-analogue of the factorial. The $b$-binomial coefficients satisfy the $q$-Pascal identity:

$$
\left(\begin{array}{c}
t-i \\
\tau
\end{array}\right)_{b}=q^{-2 i \tau}\left(\begin{array}{l}
t \\
\tau
\end{array}\right)_{b}+\left(\begin{array}{c}
t \\
\tau+i
\end{array}\right)_{b}=\left(\begin{array}{c}
t \\
\tau
\end{array}\right)_{b}+q^{2 i(\tau-t+i)}\left(\begin{array}{c}
t \\
\tau+i
\end{array}\right)_{b}
$$

which can be easily verified with the help of (3.3).

Using the $b$-binomial coefficients and the integral representation (3.31) of $g_{b}(x)$, we derive the quantum exponential relation:

$$
\begin{aligned}
g_{b}(u+v) & \stackrel{(3.31)}{=} b \int_{\mathbb{R}} d t(u+v)^{i t} \frac{e^{-\pi i b^{2} t^{2}}}{G_{b}(Q+i b t)} \stackrel{\text { B.4 })}{=} b^{2} \int_{\mathbb{R}} d t d \tau\left(\begin{array}{l}
t \\
\tau
\end{array}\right)_{b} \frac{e^{-\pi i b^{2} t^{2}}}{G_{b}(Q+i b t)} u^{i(t-\tau)} v^{i \tau} \\
& \stackrel{(B .5)}{=} b^{2} \int_{\mathbb{R}} d t d \tau \frac{e^{-\pi i b^{2}(t-\tau)^{2}-\pi i b^{2} \tau^{2}}}{G_{b}(Q+i b(t-\tau)) G_{b}(Q+i b \tau)} u^{i(t-\tau)} v^{i \tau} \\
& =b \int_{\mathbb{R}} d T \frac{e^{-\pi i b^{2} T^{2}}}{G_{b}(Q+i b T)} u^{i T} b \int_{\mathbb{R}} d \tau \frac{e^{-\pi i b^{2} \tau^{2}}}{G_{b}(Q+i b \tau)} v^{i \tau} \stackrel{\text { (.31) }}{=} g_{b}(u) g_{b}(v) .
\end{aligned}
$$

This completes the proof of Lemma 6 .

Remark 5. - After this manuscript was written we were informed that a different proof of the quantum exponential relation and of relation $(3.16)$ is given in $[\mathbb{V}]$.

Acknowledgments. - We are grateful to L.Faddeev for useful comments. We thank A.Volkov for providing us with a draft version of $[\mathrm{V}]$ prior to its publication. A.B. was supported by Alexander von Humboldt Foundation. J.T. was supported by DFG SFB 288. A part of this work was carried out during A.B.'s visit to the Department of Mathematics, University of York.

\section{References}

[Ba] E.W. Barnes: Theory of the double gamma function, Phil. Trans. Roy. Soc. A196 (1901) 265-388

[F1] L.D. Faddeev: Discrete Heisenberg-Weyl group and modular group, Lett. Math. Phys. 34 (1995) 249-254 [hep-th/9504111]

[F2] L.D. Faddeev: Modular double of a quantum group, Math. Phys. Stud. 21 (2000) 149156 [math.QA/9912078]

[FK1] L.D. Faddeev and R.M. Kashaev: Quantum dilogarithm, Mod. Phys. Lett. A9 (1994) 427-434 [hep-th/9310070]

[FKV] L.D. Faddeev, R.M. Kashaev and A.Yu. Volkov: Strongly coupled quantum discrete Liouville theory. I: Algebraic approach and duality, Comm. Math. Phys. 219 (2001) 199-219 [hep-th/0006156]

[FK2] L. Faddeev, R. Kashaev: Strongly coupled quantum discrete Liouville Theory. II: Geometric interpretation of the evolution operator, J. Phys. A35 (2002) 4043-4048 [hepth/0201049]

[FV] L.D. Faddeev and A.Yu. Volkov: Abelian current algebra and the Virasoro algebra on the lattice, Phys. Lett. B315 (1993) 311-318 [hep-th/9307048] 
[Ka] R.M. Kashaev: The non-compact quantum dilogarithm and the Baxter equations, J. Stat. Phys. 102 (2001) 923-936

[KLS] S. Kharchev, D. Lebedev and M. Semenov-Tian-Shansky: Unitary representations of $\mathscr{U}_{q}(\mathfrak{s l}(2, \mathbb{R}))$, the modular double, and the multiparticle q-deformed Toda chains, Comm. Math. Phys. 225 (2002) 573-609 [hep-th/0102180]

[KV] K. Kustermans, S.Vaes: The operator algebra approach to quantum groups, Proc. Natl. Acad. Sci. USA. 97 (2) (2000), 547-552

[PT1] B. Ponsot and J. Teschner: Liouville bootstrap via harmonic analysis on a non-compact quantum group, hep-th/9911110]

[PT2] B. Ponsot and J. Teschner: Clebsch-Gordan and Racah-Wigner coefficients for a continuous series of representations of $\mathscr{U}_{q}(\mathfrak{s l}(2, \mathbb{R}))$, Comm. Math. Phys. 224 (2001) 613-655 [math.QA/0007097]

[S] K. Schmüdgen: Operator representations of $U_{q}(\mathfrak{s l}(2, \mathbb{R}))$, Lett. Math. Phys. 37 (1996) 211-222

[Sch] M.P. Schützenberger: Une interprètation de certaines solutions de l'èquation fonctionnelle: $F(x+y)=F(x) F(y)$, C. R. Acad. Sci. Paris 236 (1953) 352-353;

J. Cigler: Operatormethoden für q-Identitäten, Monatsh. Math. 88 (1979) 87-105

[Sh] T. Shintani: On a Kronecker limit formula for real quadratic fields, J. Fac. Sci. Univ. Tokyo Sect. 1A Math. 24 (1977) 167-199

[T] J. Teschner: Liouville theory revisited, Class. Quant. Grav. 18 (2001) R153-R222 [hepth/0104158]

[W1] S. Woronowicz: Unbounded elements affiliated with $C^{*}$-algebras and non-compact quantum groups, Comm. Math. Phys. 136 (1991) 399-432

[W2] S.L. Woronowicz: Quantum exponential function, Rev. Math. Phys. 12 (2000) 873-920

[V] A.Yu. Volkov: Noncommutative hypergeometry, to appear.

A.G. Bytsko And J. Teschner, Institut für theoretische Physik, Freie Universität Berlin, Arnimallee 14, 14195 Berlin, Germany - E-mail : bytsko@physik.fu-berlin.de, teschner@physik.fu-berlin.de 